Intuitionistic fuzzy theory and its application in economy, technology and management

\title{
AN INCLUSION COMPARISON APPROACH FOR MULTIPLE CRITERIA DECISION ANALYSIS BASED ON INTERVAL-VALUED INTUITIONISTIC FUZZY SETS
}

\author{
Ting-Yu CHEN \\ Department of Industrial and Business Management, \\ Graduate Institute of Business and Management, College of Management, \\ Chang Gung University 259, Wen-Hwa 1st Road, Kwei-Shan, Taoyuan 33302, Taiwan
}

Received 11 November 2012; accepted 06 October 2013

\begin{abstract}
The theory of interval-valued intuitionistic fuzzy sets provides an intuitive and feasible way of addressing uncertain and ambiguous properties. Many useful models and methods have been developed for multiple criteria decision analysis within the interval-valued intuitionistic fuzzy environment. In contrast to the elaborate existing methods, this paper establishes a simple and effective method for managing the sophisticated data expressed by interval-valued intuitionistic fuzzy sets. An inclusion comparison possibility defined on interval-valued intuitionistic fuzzy sets is proposed, and some important properties are investigated. Then, an inclusion-based index that considers positive and negative ideals is offered. Considering the maximal comprehensive inclusion-based indices, this paper constructs a linear programming model (for consistent information) and an integrated, nonlinear programming model (for inconsistent information) to estimate the criterion weights and the optimal ranking order of the alternatives under an incomplete preference structure. The feasibility of the proposed method is illustrated by a practical example of selecting a suitable bridge construction method, and a comparative analysis with other relevant methods is conducted to validate the effectiveness and applicability of the proposed methodology.
\end{abstract}

Keywords: interval-valued intuitionistic fuzzy set, multiple criteria decision analysis, inclusion comparison possibility, inclusion-based index, incomplete preference structure.

JEL Classification: C44, D81, R42.

\section{Introduction}

Multiple criteria decision analysis (MCDA) problems are common. Because uncertainty always exists, modeling uncertainty in subjective human management becomes increasingly important in decision analysis. The theory of fuzzy sets is a useful way to manage uncertainty 
or imprecision arising from mental phenomena in decision making. However, in reality, the evaluation of membership values may not always be certain. There may be some degree of hesitation between membership and non-membership. Intuitionistic fuzzy sets (IFSs), which were introduced by Atanassov $(1983,1986)$, are appropriate for addressing the problem of insufficient information. Importantly, IFSs are mathematically equivalent to interval-valued fuzzy sets (Deschrijver, Kerre 2007). Nevertheless, IFSs and interval-valued fuzzy sets are different because they are based on different semantics and have distinct backgrounds and practical applicability (Atanassov 2005; Dubois et al. 2005; Deschrijver, Kerre 2007; Li 2012a). Li (2005) first developed MCDA models and methods using IFSs. He introduced intuitionistic fuzzy MCDA problems and constructed several linear programming models to generate optimal criteria weights. At present, the IFS theory has been successfully applied to MCDA and group decision-making problems (Li 2010a; Li et al. 2010; Zhang, Liu 2010; Li 2011a; Wei et al. 2012). Atanassov and Gargov (1989) introduced the concept of interval-valued intuitionistic fuzzy sets (IVIFSs), which are a generalization of IFSs. IVIFSs are characterized by membership and non-membership functions whose values are intervals rather than exact numbers. $\mathrm{Li}$ (2012b) developed the representation theorem and extension principles for IFSs. $\mathrm{Li}$ (2011b) proposed the representation theorem of IVIFSs using the concept of level sets. In addition, the algebraic operations over IVIFSs were defined based on the extension principle. IVIFSs have received more attention because of their superior ability to handle imprecise and ambiguous information in real-world applications.

IVIFSs have been widely used in the MCDA field, and substantial research has been devoted to enriching the development of decision-making models and methods within the IVIFS environment. Li (2010b) developed a nonlinear programming methodology, based on the technique for order preference by similarity to ideal solution (TOPSIS), to solve MCDA problems using ratings of alternatives together with the criterion weights expressed by IVIFSs. Li (2010c) proposed a mathematical-programming methodology of matrix games with payoffs represented by IVIFSs. Nayagam et al. (2011) introduced a new accuracy function and proposed an MCDA method based on IVIFSs. Chen and Yang (2011) established optimization models to determine the criterion weights under incomplete information and developed group decision-making methods for IVIFS settings. Chen et al. (2011) proposed a group decision-making method based on interval-valued intuitionistic fuzzy preference relation matrices and aggregation operators. Ye (2012) developed the concept of the reduct IFSs of IVIFSs with respect to adjustable weight vectors; this author also developed the Dice similarity measure, based on the reduct IFSs, to explore the effects of optimism, neutralism, and pessimism in decision making. Combining the interval-valued intuitionistic fuzzy geometric aggregation operator with the Choquet integral-based Hamming distance, Tan (2011) developed an extension of the TOPSIS method for solving group decision-making problems. Park et al. (2011) also extended the TOPSIS method to solve multiple criteria group decision-making problems within the IVIFS environment. Based on the concept of relative closeness coefficients, Li (2011c) developed auxiliary nonlinear programming models to solve MCDA problems with IVIFSs. Wei et al. (2011) introduced a correlation and correlation coefficients for IVIFSs and then established an optimization model, based on the negative ideal solution and a max-min operator, to solve MCDA problems. Based on 
the concepts of relative closeness coefficients and weighted Euclidean distances, Wang et al. (2013) constructed a pair of nonlinear programming models for MCDA using IVIFSs. Wei and Zhao (2012) introduced the induced intuitionistic fuzzy correlated averaging operator and the induced intuitionistic fuzzy correlated geometric operator to develop an MCDA method based on IVIFSs. Chen and Yang (2012) considered the risk attitude of the decision maker to define a new class of decision functions and to solve MCDA problems with interval-valued intuitionistic fuzzy information.

$\mathrm{Li}$ (2011c) first proposed the concept of an inclusion comparison probability to compare the closeness IFSs. Li (2010d) defined the likelihood that one alternative is not inferior to another. Next, he constructed a likelihood matrix and determined the optimal degrees of membership for ranking the alternatives. Wang et al. (2013) also used the concept of interval number likelihood to generate a ranking order of alternatives. Li (2011c) extended the likelihood measure to define an inclusion comparison probability, allowing comparisons between alternatives. Similar to Szmidt and Kacprzyk (2001), Li (2011c) regarded the inclusion relation between the closeness IFSs as an intuitionistic fuzzy event from a probability viewpoint. He then defined the inclusion comparison probability and investigated its useful and other important properties.

MCDA methods with IVIFSs have been extensively studied in the past. However, little research has utilized the inclusion comparison-based approach (e.g., the employment of likelihood measures or inclusion comparison probabilities) to handle interval-valued intuitionistic fuzzy MCDA problems. That is, in regard to the existing MCDA techniques with IVIFSs, the inclusion comparison-based approach has not been the core of decision-making methodologies. Although Li (2011c) utilized the concept of inclusion comparison probabilities in his proposed closeness coefficient-based nonlinear programming method, his developed inclusion comparison probability is still defined upon IFSs, not IVIFSs. Considering the advantages of the inclusion comparison probability on IFSs, this paper extends the concept to propose an inclusion comparison possibility within the interval-valued intuitionistic fuzzy decision environment. This paper presents the concept of the lower and upper inclusion comparison possibilities defined on IVIFSs and then determines an interval-valued intuitionistic fuzzy inclusion comparison possibility. Additionally, we propose a novel way to incorporate inclusion comparison possibilities into the core structure of TOPSIS, which is new and different from those methods that have been previously developed.

The purpose of this paper is to develop a novel interval-valued intuitionistic fuzzy MCDA method by using inclusion comparison possibilities defined on IVIFSs. In a manner similar to TOPSIS, an inclusion-based index that considers positive and negative ideals is proposed. In view of diversiform preference types, we represent the various forms of preference structures of the decision maker. Next, we advance an inclusion comparison possibility approach to develop a new MCDA method, including programming models for assessing criterion weights with incomplete information and for handling decision-making problems in the IVIFS context.

This article is organized as follows: Section 1 briefly reviews concepts of IVIFSs and formulates an MCDA problem with IVIFS data. Section 2 proposes inclusion comparison possibilities for the IVIFS environment and investigates several important properties of the proposed measures. Section 3 proposes the concept of the inclusion-based index and develops 
an MCDA method using an inclusion comparison possibility approach for managing interval-valued intuitionistic fuzzy decision making with incomplete preference information. In addition, this section constructs linear and nonlinear programming models for consistent and inconsistent preference structures, respectively, to determine the criterion weights under limited weight information. Section 4 examines the feasibility and applicability of the proposed method by illustrating how it can be applied to a selection problem for bridge construction methods. This section also provides a comparative analysis with the widely used fuzzy TOPSIS method and the closeness coefficient-based nonlinear programming method (Li 2011c). Finally, the last Section presents our conclusions.

\section{Decision environment on IVIFSs}

Before formulating a decision environment based on IVIFSs, we first briefly review the basic concept of IVIFSs.

Definition 1. Let Int $([0,1])$ denote the set of all closed subintervals of $[0,1]$. Let $X$ be an ordinary finite, nonempty set. An IVIFS $\tilde{A}$ in $X$ is defined as:

$$
\tilde{A}=\left\{\left\langle x, \mu_{\tilde{A}}(x), v_{\tilde{A}}(x)\right\rangle \mid x \in X\right\},
$$

where the functions $\mu_{\tilde{A}}: X \rightarrow \operatorname{Int}([0,1])\left(x \in X \rightarrow \mu_{\tilde{A}}(x) \subseteq[0,1]\right)$ and $v_{\tilde{A}}: X \rightarrow \operatorname{Int}([0,1])$ $\left(x \in X \rightarrow v_{\tilde{A}}(x) \subseteq[0,1]\right)$ define the intervals of the degree of membership and the degree of non-membership of the element $x \in X$ to the set $\tilde{A}$, respectively, and for every $x \in X$, $0 \leq \sup \left\{\mu_{\tilde{A}}(x)\right\}+\sup \left\{v_{\tilde{A}}(x)\right\} \leq 1$.

Definition 2. For every IVIFS $\tilde{A}$ in $X, \mu_{\tilde{A}}(x)$ and $\nu_{\tilde{A}}(x)$ are closed intervals rather than real numbers, and their lower and upper boundaries are denoted by $\mu_{\tilde{\tilde{A}}}(x), \mu_{\tilde{A}}^{+}(x), v_{\tilde{A}}(x)$, and $v_{\tilde{A}}^{+}(x)$, respectively. Then, $\tilde{A}$ can be expressed as follows:

$$
\tilde{A}=\left\{\left\langle x,\left[\mu_{\tilde{\tilde{A}}}(x), \mu_{\tilde{A}}^{+}(x)\right],\left[v_{\tilde{\tilde{A}}}^{-}(x), v_{\tilde{A}}^{+}(x)\right]\right\rangle \mid x \in X\right\},
$$

where the expression is subject to the condition $0 \leq \mu_{\tilde{A}}^{+}(x)+v_{\tilde{A}}^{+}(x) \leq 1$.

For every $x \in X$, the hesitation interval relative to $\tilde{A}$ is computed as:

$$
\pi_{\tilde{A}}(x)=\left[\pi_{\tilde{A}}(x), \pi_{\tilde{A}}^{+}(x)\right]=\left[1-\mu_{\tilde{A}}^{+}(x)-v_{\tilde{A}}^{+}(x), 1-\mu_{\tilde{A}}(x)-v_{\tilde{A}}(x)\right] .
$$

If $\mu_{\tilde{A}}(x)=\mu_{\tilde{A}}(x)=\mu_{\tilde{A}}^{+}(x)$ and $v_{\tilde{A}}(x)=v_{\tilde{A}}(x)=v_{\tilde{A}}^{+}(x)$, then the given IVIFS $\tilde{A}$ is reduced to an ordinary IFS. For convenience of notation, the set of all IVIFSs in $X$ is denoted by IVIFS(X). Let $\tilde{A}_{x}$ denote a value of IVIFS $\tilde{A}$ where:

$$
\tilde{A}_{x}=\left(\mu_{\tilde{A}}(x), v_{\tilde{A}}(x)\right)=\left(\left[\mu_{\tilde{\tilde{A}}}(x), \mu_{\tilde{A}}^{+}(x)\right],\left[v_{\tilde{\tilde{A}}}^{\bar{c}}(x), v_{\tilde{A}}^{+}(x)\right]\right) .
$$

To consider an MCDA problem, the method of evaluating alternatives and making decisions is guided by the subjective judgments of the decision maker. Thus, the ratings of alternative evaluations used in MCDA can be reasonably considered to be expressed as IVIFSs. Let $A_{1}, A_{2}, \cdots$, and $A_{m}$ represent the non-dominated alternatives from which a decision maker must choose, where $\mathrm{m}$ represents the number of alternatives, and let $A\left(=\left\{A_{1}, A_{2}, \cdots, A_{m}\right\}\right)$ 
be an alternative set. Let $x_{1}, x_{2}, \ldots$, and $x_{n}$ represent the criteria with which the alternative performances are measured, where n represents the number of criteria. Moreover, denote $X$ $\left(=\left\{x_{1}, x_{2}, \cdots, x_{n}\right\}\right)$ as a criterion set. In general, the criterion set $X$ can be divided into two sets, $X_{b}$ and $X_{c}$, where $X_{b}$ denotes a collection of benefit criteria, $X_{c}$ denotes a collection of cost criteria, $X_{b} \cap X_{c}=\varnothing$, and $X_{b} \cup X_{c}=X$.

Let $\tilde{A}_{i j}$ denote the evaluation of the alternative $A_{i} \in A(\mathrm{i}=1,2, \cdots, \mathrm{m})$ with respect to the criterion $x_{j} \in X(j=1,2, \ldots, n)$. Then, $\tilde{A}_{i j}$ can be expressed as the following:

$$
\tilde{A}_{i j}=\left(\mu_{i j}, v_{i j}\right)=\left(\left[\mu_{i j}^{-}, \mu_{i j}^{+}\right],\left[v_{i j}^{-}, v_{i j}^{+}\right]\right),
$$

where $\mu_{i j}\left(=\left[\mu_{i j}^{-}, \mu_{i j}^{+}\right]\right)$represents the interval of the membership degree for which alternative $A_{i}$ satisfies criterion $x_{j}$ and $v_{i j}\left(=\left[v_{i j}^{-}, v_{i j}^{+}\right]\right)$indicates the interval of the non-membership degree for which alternative $A_{i}$ does not satisfy criterion $x_{j}$ given by the decision maker. For every $A_{i} \in A$ and $x_{j} \in X$, the hesitation interval of $\tilde{A}_{i j}$ is computed as follows:

$$
\pi_{i j}=\left[\pi_{i j}^{-}, \pi_{i j}^{+}\right]=\left[1-\mu_{i j}^{+}-v_{i j}^{+}, 1-\mu_{i j}^{-}-v_{i j}^{-}\right] .
$$

The characteristics of the alternative $A_{i}$ can be represented by the IVIFS in the following manner:

$$
\begin{aligned}
\tilde{A}_{i}= & \left\{\left\langle x_{1},\left(\mu_{i 1}, v_{i 1}\right)\right\rangle,\left\langle x_{2},\left(\mu_{i 2}, v_{i 2}\right)\right\rangle, \cdots,\left\langle x_{n},\left(\mu_{i n}, v_{i n}\right)\right\rangle\right\}= \\
& \left\{\left\langle x_{j},\left(\left[\mu_{i j}^{-}, \mu_{i j}^{+}\right],\left[v_{i j}^{-}, v_{i j}^{+}\right]\right)\right\rangle \mid x_{j} \in X, j=1,2, \cdots, n\right\}, i=1,2, \cdots, m .
\end{aligned}
$$

An interval-valued intuitionistic fuzzy decision matrix $\tilde{D}$ is expressed as follows:

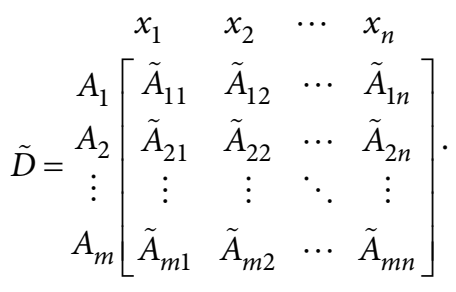

\section{Inclusion comparison possibility on IVIFSs}

To make a comparison between IVIFSs, we extended the inclusion comparison probability introduced by $\mathrm{Li}$ (2011c) to the interval-valued intuitionistic fuzzy decision environment. Let $A_{\rho}$ and $A_{\rho^{\prime}}$ be two alternatives in the alternative set $A$. Denote $\tilde{A}_{\rho j}$ and $\tilde{A}_{\rho^{\prime} j}$ as the evaluation values of alternatives $A_{\rho}$ and $A_{\rho^{\prime}}$, respectively, with respect to criterion $x_{j}$, and:

$$
\begin{gathered}
\tilde{A}_{\rho j}=\left(\mu_{\rho j}, v_{\rho j}\right)=\left(\left[\mu_{\rho j}^{-}, \mu_{\rho j}^{+}\right],\left[v_{\rho j}^{-}, v_{\rho j}^{+}\right]\right), \\
\tilde{A}_{\rho^{\prime} j}=\left(\mu_{\rho^{\prime} j}, v_{\rho^{\prime} j}\right)=\left(\left[\mu_{\rho^{\prime} j}^{-}, \mu_{\rho^{\prime} j}^{+}\right],\left[v_{\rho^{\prime} j}^{-}, v_{\rho^{\prime} j}^{+}\right]\right) .
\end{gathered}
$$

The binary relation of “ $\tilde{A}_{\rho j} \supseteq \tilde{A}_{\rho^{\prime} j}$ ” is equivalently written as $\mu_{\rho j}^{-} \geq \mu_{\rho^{\prime} j}^{-}, \mu_{\rho j}^{+} \geq \mu_{\rho^{\prime} j}^{+}$, $v_{\rho j}^{-} \leq v_{\rho^{\prime} j}^{-}$, and $v_{\rho j}^{+} \leq v_{\rho^{\prime} j}^{+}$according to the inclusion relation of IVIFSs. Considering 
“ $\tilde{A}_{\rho j} \supseteq \tilde{A}_{\rho^{\prime} j}$ ” as an interval-valued intuitionistic fuzzy event, we can compare $A_{\rho j}$ and $A_{\rho^{\prime} j}$ by computing the possibility of the inclusion comparison event of $\tilde{A}_{\rho j}$ and $\tilde{A}_{\rho^{\prime} j}$. The possibility of the interval-valued intuitionistic fuzzy event “ $\tilde{A}_{\rho j} \supseteq \tilde{A}_{\rho^{\prime} j}$ ”, denoted by $p\left(\tilde{A}_{\rho j} \supseteq \tilde{A}_{\rho^{\prime} j}\right)$, is called the inclusion comparison possibility of $\tilde{A}_{\rho j}$ and $\tilde{A}_{\rho^{\prime} j}$. We determine $p\left(\tilde{A}_{\rho j} \supseteq \tilde{A}_{\rho^{\prime} j}\right)$ by using the lower and upper inclusion comparison possibilities $p^{-}\left(\tilde{A}_{\rho j} \supseteq \tilde{A}_{\rho^{\prime} j}\right)$ and $p^{+}\left(\tilde{A}_{\rho j} \supseteq \tilde{A}_{\rho^{\prime} j}\right)$, respectively, of $\tilde{A}_{\rho j}$ and $\tilde{A}_{\rho^{\prime} j}$.

Definition 3. Let $\tilde{A}_{\rho j}=\left(\left[\mu_{\rho j}^{-}, \mu_{\rho j}^{+}\right],\left[v_{\rho j}^{-}, v_{\rho j}^{+}\right]\right)$and $\tilde{A}_{\rho^{\prime} j}=\left(\left[\mu_{\rho^{\prime} j}^{-}, \mu_{\rho^{\prime} j}^{+}\right],\left[v_{\rho^{\prime} j}^{-}, v_{\rho^{\prime} j}^{+}\right]\right)$be any two values of IVIFSs defined on $X$, where $0 \leq \mu_{\rho j}^{+}+v_{\rho j}^{+} \leq 1$ and $0 \leq \mu_{\rho^{\prime} j}^{+}+v_{\rho^{\prime} j}^{+} \leq 1$. The lower inclusion comparison possibility $p^{-}\left(\tilde{A}_{\rho j} \supseteq \tilde{A}_{\rho^{\prime} j}\right)$ of $\tilde{A}_{\rho j}$ and $\tilde{A}_{\rho^{\prime} j}$ is defined as:

$$
p^{-}\left(\tilde{A}_{\rho j} \supseteq \tilde{A}_{\rho^{\prime} j}\right)=\max \left\{1-\max \left\{\frac{\left(1-v_{\rho^{\prime} j}^{-}\right)-\mu_{\rho j}^{-}}{\left(1-\mu_{\rho j}^{-}-v_{\rho j}^{+}\right)+\left(1-\mu_{\rho^{\prime} j}^{+}-v_{\rho^{\prime} j}^{-}\right)}, 0\right\}, 0\right\} .
$$

The upper inclusion comparison possibility $p^{+}\left(\tilde{A}_{\rho j} \supseteq \tilde{A}_{\rho^{\prime} j}\right)$ is defined as:

$$
p^{+}\left(\tilde{A}_{\rho j} \supseteq \tilde{A}_{\rho^{\prime} j}\right)=\max \left\{1-\max \left\{\frac{\left(1-v_{\rho^{\prime} j}^{+}\right)-\mu_{\rho j}^{+}}{\left(1-\mu_{\rho j}^{+}-v_{\rho j}^{-}\right)+\left(1-\mu_{\rho^{\prime} j}^{-}-v_{\rho^{\prime} j}^{+}\right)}, 0\right\}, 0\right\} .
$$

Property 1. Let $\tilde{A}_{\rho j}=\left(\left[\mu_{\rho j}^{-}, \mu_{\rho j}^{+}\right],\left[v_{\rho j}^{-}, v_{\rho j}^{+}\right]\right)$and $\tilde{A}_{\rho^{\prime} j}=\left(\left[\mu_{\rho^{\prime} j}^{-}, \mu_{\rho^{\prime} j}^{+}\right],\left[v_{\rho^{\prime} j}^{-}, v_{\rho^{\prime} j}^{+}\right]\right)$be any two values of IVIFSs defined on $\mathrm{X}$. The lower and upper inclusion comparison possibilities $p^{-}\left(\tilde{A}_{\rho j} \supseteq \tilde{A}_{\rho^{\prime} j}\right)$ and $p^{+}\left(\tilde{A}_{\rho j} \supseteq \tilde{A}_{\rho^{\prime} j}\right)$, respectively, of $\tilde{A}_{\rho j}$ and $\tilde{A}_{\rho^{\prime} j}$ satisfy the following properties:

(P1.1) $0 \leq p^{-}\left(\tilde{A}_{\rho j} \supseteq \tilde{A}_{\rho^{\prime} j}\right) \leq 1$;

(P1.2) $0 \leq p^{+}\left(\tilde{A}_{\rho j} \supseteq \tilde{A}_{\rho^{\prime} j}\right) \leq 1$;

(P1.3) $p^{-}\left(\tilde{A}_{\rho j} \supseteq \tilde{A}_{\rho^{\prime} j}\right) \leq p^{+}\left(\tilde{A}_{\rho j} \supseteq \tilde{A}_{\rho^{\prime} j}\right)$;

(P1.4) $p^{-}\left(\tilde{A}_{\rho j} \supseteq \tilde{A}_{\rho^{\prime} j}\right)+p^{+}\left(\tilde{A}_{\rho^{\prime} j} \supseteq \tilde{A}_{\rho j}\right)=1$.

Proof. See Appendix I.

Definition 4. Let $\tilde{A}_{\rho j}=\left(\left[\mu_{\rho j}^{-}, \mu_{\rho j}^{+}\right],\left[v_{\rho j}^{-}, v_{\rho j}^{+}\right]\right)$and $\tilde{A}_{\rho^{\prime} j}=\left(\left[\mu_{\rho^{\prime} j}^{-}, \mu_{\rho^{\prime} j}^{+}\right],\left[v_{\rho^{\prime} j}^{-}, v_{\rho^{\prime} j}^{+}\right]\right)$be any two values of IVIFSs defined on X. The inclusion comparison possibility $p\left(\tilde{A}_{\rho j} \supseteq \tilde{A}_{\rho^{\prime} j}\right)$ of $\tilde{A}_{\rho j}$ and $\tilde{A}_{\rho^{\prime} j}$ is defined as follows:

$$
p\left(\tilde{A}_{\rho j} \supseteq \tilde{A}_{\rho^{\prime} j}\right)=\frac{1}{2}\left(p^{-}\left(\tilde{A}_{\rho j} \supseteq \tilde{A}_{\rho^{\prime} j}\right)+p^{+}\left(\tilde{A}_{\rho j} \supseteq \tilde{A}_{\rho^{\prime} j}\right)\right) .
$$

That is, $\tilde{A}_{\rho j}$ is not smaller than $\tilde{A}_{\rho^{\prime} j}$ to the degree of $p\left(\tilde{A}_{\rho j} \supseteq \tilde{A}_{\rho^{\prime} j}\right)$. 
Property 2. Let $\tilde{A}_{\rho j}=\left(\left[\mu_{\rho j}^{-}, \mu_{\rho j}^{+}\right],\left[v_{\rho j}^{-}, v_{\rho j}^{+}\right]\right)$and $\tilde{A}_{\rho^{\prime} j}=\left(\left[\mu_{\rho^{\prime} j}^{-}, \mu_{\rho^{\prime} j}^{+}\right],\left[v_{\rho^{\prime} j}^{-}, v_{\rho^{\prime} j}^{+}\right]\right)$be any two values of IVIFSs defined on $X$. The inclusion comparison possibility $p\left(\tilde{A}_{\rho j} \supseteq \tilde{A}_{\rho^{\prime} j}\right)$ of $\tilde{A}_{\rho j}$ and $\tilde{A}_{\rho^{\prime} j}$ satisfies the following properties:

(P2.1) $0 \leq p\left(\tilde{A}_{\rho j} \supseteq \tilde{A}_{\rho^{\prime} j}\right) \leq 1$;

(P2.2) $p\left(\tilde{A}_{\rho j} \supseteq \tilde{A}_{\rho^{\prime} j}\right)=0$ if $1-v_{\rho j}^{-} \leq \mu_{\rho^{\prime} j}^{-}$;

(P2.3) $p\left(\tilde{A}_{\rho j} \supseteq \tilde{A}_{\rho^{\prime} j}\right)=1$ if $\mu_{\rho j}^{-} \geq 1-v_{\rho^{\prime} j}^{-}$;

(P2.4) $p\left(\tilde{A}_{\rho j} \supseteq \tilde{A}_{\rho^{\prime} j}\right)+p\left(\tilde{A}_{\rho j} \subseteq \tilde{A}_{\rho^{\prime} j}\right)=1$;

(P2.5) $p\left(\tilde{A}_{\rho j} \supseteq \tilde{A}_{\rho^{\prime} j}\right)=p\left(\tilde{A}_{\rho j} \subseteq \tilde{A}_{\rho^{\prime} j}\right)=0.5$ if $p\left(\tilde{A}_{\rho j} \supseteq \tilde{A}_{\rho^{\prime} j}\right)=p\left(\tilde{A}_{\rho j} \subseteq \tilde{A}_{\rho^{\prime} j}\right)$;

(P2.6) $p\left(\tilde{A}_{\rho j} \supseteq \tilde{A}_{\rho^{\prime} j}\right) \geq 0.5$ if $p\left(\tilde{A}_{\rho j} \supseteq \tilde{A}_{\rho^{\prime \prime} j}\right) \geq 0.5$ and $p\left(\tilde{A}_{\rho^{\prime \prime} j} \supseteq \tilde{A}_{\rho^{\prime} j}\right) \geq 0.5$.

Proof. See Appendix II.

If $\tilde{A}_{\rho j}$ and $\tilde{A}_{\rho^{\prime} j}$ reduce to IFSs, the inclusion comparison possibility of $\tilde{A}_{\rho j}$ and $\tilde{A}_{\rho^{\prime} j}$ becomes the following:

$$
p\left(\tilde{A}_{\rho j} \supseteq \tilde{A}_{\rho^{\prime} j}\right)=\max \left\{1-\max \left\{\frac{\left(1-v_{\rho^{\prime} j}\right)-\mu_{\rho j}}{\left(1-\mu_{\rho j}-v_{\rho j}\right)+\left(1-\mu_{\rho^{\prime} j}-v_{\rho^{\prime} j}\right)}, 0\right\}, 0\right\} .
$$

The above formula is the same as the definition provided by $\mathrm{Li}$ (2011c).

\section{MCDA method with inclusion comparison possibilities}

This section applies the concept of inclusion comparison possibilities to develop a new MCDA method within the IVIFS environment under incomplete preference information. Anchor dependency is common in decision making: when the decision maker must make assessments, he/she tends to anchor the judgments to points of reference. Thus, the concepts of positive-ideal and negative-ideal solutions and their displacements facilitate the rationale underlying human decision-making processes. The displacement of positive and negative ideals may affect the contrast of currently achievable performance among alternatives. Accordingly, it is necessary to incorporate displaced ideals and anchor dependency into decision analysis. In this paper, we utilize the concept of inclusion comparison possibilities to propose an inclusion-based index considering both positive and negative ideals.

\subsection{The proposed method}

Regarding the MCDA problem based on IVIFSs, recall that $X_{b}$ and $X_{c}$ are collections of benefit and cost criteria, respectively. The interval-valued intuitionistic fuzzy positive-ideal solution, denoted as $\tilde{A}_{*}$, is defined as follows: 


$$
\begin{gathered}
\tilde{A}_{*}=\left\{\left\langle x_{1},\left(\mu_{\star_{1}}, \nu_{\star_{1}}\right)\right\rangle,\left\langle x_{2},\left(\mu_{\star_{2}}, \nu_{\star_{2}}\right)\right\rangle, \cdots,\left\langle x_{n},\left(\mu_{\star_{n}}, \nu_{\star_{n}}\right)\right\rangle\right\}= \\
\left\{\left\langle x_{j},\left(\left[\mu_{\star_{j}}^{-}, \mu_{\star_{j}}^{+}\right],\left[\nu_{\star_{j}}^{-}, \nu_{\star_{j}}^{+}\right]\right)\right\rangle \mid x_{j} \in X, j=1,2, \cdots, n\right\},
\end{gathered}
$$

where

$$
\begin{aligned}
{\left[\mu_{\star_{j}}^{-}, \mu_{\star_{j}}^{+}\right]=} & {\left[\left(\left(\max _{i} \mu_{i j}^{-} \mid x_{j} \in X_{b}\right),\left(\min _{i} \mu_{i j}^{-} \mid x_{j} \in X_{c}\right)\right),\right.} \\
& \left.\left(\left(\max _{i} \mu_{i j}^{+} \mid x_{j} \in X_{b}\right),\left(\min _{i} \mu_{i j}^{+} \mid x_{j} \in X_{c}\right)\right)\right], \\
{\left[v_{\star_{j}}^{-}, v_{\star_{j}}^{+}\right]=} & {\left[\left(\left(\min _{i} v_{i j}^{-} \mid x_{j} \in X_{b}\right),\left(\max _{i} v_{i j}^{-} \mid x_{j} \in X_{c}\right)\right),\right.} \\
& \left.\left(\left(\min _{i} v_{i j}^{+} \mid x_{j} \in X_{b}\right),\left(\max _{i} v_{i j}^{+} \mid x_{j} \in X_{c}\right)\right)\right] .
\end{aligned}
$$

For convenience, let $\mu_{\star_{j}}\left(=\left[\mu_{\star_{j}}^{-}, \mu_{\star_{j}}^{+}\right]\right)$and $v_{\star_{j}}\left(=\left[\nu_{\star_{j}}^{-}, \nu_{\star_{j}}^{+}\right]\right)$. Then, the evaluation of the positive-ideal solution with respect to $x_{j}$ is given by:

$$
\tilde{A}_{\star_{j}}=\left(\mu_{\star_{j}}, \nu_{\star_{j}}\right)=\left(\left[\mu_{\star_{j}}^{-}, \mu_{\star_{j}}^{+}\right],\left[\nu_{\star_{j}}^{-}, \nu_{\star_{j}}^{+}\right]\right) .
$$

The interval-valued intuitionistic fuzzy negative-ideal solution, denoted as $\tilde{A}_{-}$, is defined as follows:

$$
\begin{gathered}
\tilde{A}_{-}=\left\{\left\langle x_{1},\left(\mu_{-1}, v_{-1}\right)\right\rangle,\left\langle x_{2},\left(\mu_{-2}, v_{-2}\right)\right\rangle, \cdots,\left\langle x_{n},\left(\mu_{-n}, v_{-n}\right)\right\rangle\right\}= \\
\left\{\left\langle x_{j},\left(\left[\mu_{-j}^{-}, \mu_{-j}^{+}\right],\left[v_{-j}^{-}, v_{-j}^{+}\right]\right)\right\rangle \mid x_{j} \in X, j=1,2, \cdots, n\right\},
\end{gathered}
$$

where

$$
\begin{aligned}
{\left[\mu_{-j}^{-}, \mu_{-j}^{+}\right]=} & {\left[\left(\left(\min _{i} \mu_{i j}^{-} \mid x_{j} \in X_{b}\right),\left(\max _{i} \mu_{i j}^{-} \mid x_{j} \in X_{c}\right)\right),\right.} \\
& \left.\left(\left(\min _{i} \mu_{i j}^{+} \mid x_{j} \in X_{b}\right),\left(\max _{i} \mu_{i j}^{+} \mid x_{j} \in X_{c}\right)\right)\right], \\
{\left[v_{-j}^{-}, v_{-j}^{+}\right]=} & {\left[\left(\left(\max _{i} v_{i j}^{-} \mid x_{j} \in X_{b}\right),\left(\min _{i} v_{i j}^{-} \mid x_{j} \in X_{c}\right)\right),\right.} \\
& \left.\left(\left(\max _{i} v_{i j}^{+} \mid x_{j} \in X_{b}\right),\left(\min _{i} v_{i j}^{+} \mid x_{j} \in X_{c}\right)\right)\right] .
\end{aligned}
$$

Let $\mu_{-j}\left(=\left[\mu_{-j}^{-}, \mu_{-j}^{+}\right]\right)$and $v_{-j}\left(=\left[v_{-j}^{-}, v_{-j}^{+}\right]\right)$for brevity. Thus, the evaluation of the negative-ideal solution in terms of $x_{j}$ is given by:

$$
\tilde{A}_{-j}=\left(\mu_{-j}, v_{-j}\right)=\left(\left[\mu_{-j}^{-}, \mu_{-j}^{+}\right],\left[v_{-j}^{-}, v_{-j}^{+}\right]\right) .
$$

To define an inclusion-based index, we first acquire the inclusion comparison possibility between evaluations of each alternative and the positive-ideal/negative-ideal solutions. For every $A_{i} \in A$ and $x_{j} \in X_{b}$, we calculate the inclusion comparison possibility $p\left(\tilde{\tilde{A}}_{\sim_{j}} \supseteq \tilde{A}_{i j}\right)$ that $\tilde{A}_{i j}$ is not larger than $\tilde{A}_{*_{j}}$ and the possibility $p\left(\tilde{A}_{i j} \supseteq \tilde{A}_{-j}\right)$ that $\tilde{A}_{i j}$ is not smaller than 
$\tilde{A}_{-j}$. The alternative $A_{i}$ produces good performance on the benefit criterion $\mathrm{x}_{\mathrm{j}}$ if the evaluation $\tilde{A}_{i j}$ has a low possibility of being inferior to $\tilde{A}_{*_{j}}$ and a high possibility of being superior to $\tilde{A}_{-j}$. In contrast, we compute $p\left(\tilde{A}_{i j} \supseteq \tilde{A}_{*_{j}}\right)$ that $\tilde{A}_{i j}$ is not smaller than $\tilde{A}_{*_{j}}$ and $p\left(\tilde{A}_{-j} \supseteq \tilde{A}_{i j}\right)$ that $\tilde{A}_{i j}$ is not larger than $\tilde{A}_{-j}$ for every $A_{i} \in A$ and $x_{j} \in X_{c}$. The alternative $\mathrm{A}_{\mathrm{i}}$ performs well on the cost criterion $x_{j}$ when the evaluation $\tilde{A}_{i j}$ has a low possibility of being farther from $\tilde{A}_{*_{j}}$ and a high possibility of being farther from $\tilde{A}_{-j}$. Following the above discussion, the inclusion-based index $I\left(\tilde{A}_{i j}\right)$ of $\tilde{A}_{i j}$ is defined as follows:

$$
I\left(\tilde{A}_{i j}\right)= \begin{cases}\frac{p\left(\tilde{A}_{i j} \supseteq \tilde{A}_{-j}\right)}{p\left(\tilde{A}_{*_{j}} \supseteq \tilde{A}_{i j}\right)+p\left(\tilde{A}_{i j} \supseteq \tilde{A}_{-j}\right)} & \text { if } x_{j} \in X_{b}, \\ \frac{p\left(\tilde{A}_{-j} \supseteq \tilde{A}_{i j}\right)}{p\left(\tilde{A}_{i j} \supseteq \tilde{A}_{*_{j}}\right)+p\left(\tilde{A}_{-j} \supseteq \tilde{A}_{i j}\right)} & \text { if } x_{j} \in X_{c},\end{cases}
$$

where $0 \leq I\left(\tilde{A}_{i j}\right) \leq 1$ for every $A_{i} \in \underset{\sim}{A}$ and $x_{j} \in X$. The higher the inclusion-based index $I\left(\tilde{A}_{i j}\right)$ is, the better the evaluation $\tilde{A}_{i j}$.

Let $w_{j}$ be the importance weight $w_{j}$ of each criterion $x_{j} \in X$ satisfying the following normalization conditions: $w_{j} \in[0,1](j=1,2, \cdots, n)$ and $\sum_{j=1}^{n} w_{j}=1$. Let $\Gamma_{0}$ denote a set of all weight vectors such that:

$$
\Gamma_{0}=\left\{\left(w_{1}, w_{2}, \cdots, w_{n}\right) \mid w_{j} \geq 0(j=1,2, \cdots, n), \sum_{j=1}^{n} w_{j}=1\right\} .
$$

Considering the importance weights of various criteria, the comprehensive inclusion-based index $\operatorname{CI}\left(\tilde{A}_{i}\right)$ of the characteristics $\tilde{A}_{i}$ for the alternative $\mathrm{A}_{\mathrm{i}}$ is:

$$
C I\left(\tilde{A}_{i}\right)=\sum_{j=1}^{n} I\left(\tilde{A}_{i j}\right) \cdot w_{j} .
$$

Because the decision maker may not be willing or able to specify his/her preferences in the detailed manner required by this theoretical methodology, unknown and incomplete (i.e., imprecise or partial) information is a likely parameter in many MCDA problems (Han, Liu 2011). In general, incomplete information about criterion weights provided by the decision maker can be constructed by using several basic ranking forms (Park 2004; Park et al. 2009, 2011; Li 2011c; Wei et al. 2011). Following the definitions proposed by Xu and Chen (2008) and $\mathrm{Li}$ (2011c), we considered five basic ranking forms of incomplete information about criterion weights:

(i) A weak ranking:

$$
\Gamma_{1}=\left\{\left(w_{1}, w_{2}, \cdots, w_{n}\right) \in \Gamma_{0} \mid w_{j_{1}} \geq w_{j_{2}} \text { for all } j_{1} \in \Upsilon_{1} \text { and } j_{2} \in \Lambda_{1}\right\},
$$

where $\Upsilon_{1}$ and $\Lambda_{1}$ are two disjoint subsets of the subscript index set $N=\{1,2, \cdots, n\}$ of all criteria.

(ii) A strict ranking:

$$
\Gamma_{2}=\left\{\left(w_{1}, w_{2}, \cdots, w_{n}\right) \in \Gamma_{0} \mid w_{j_{1}}-w_{j_{2}} \geq \delta_{j_{1} j_{2}} \text { for all } j_{1} \in \Upsilon_{2} \text { and } j_{2} \in \Lambda_{2}\right\},
$$


where $\delta_{j_{1} j_{2}}$ is a constant that satisfies the condition $\delta_{j_{1} j_{2}}>0$, and $\Upsilon_{2}$ and $\Lambda_{2}$ are two disjoint subsets of $N$.

(iii) A ranking of differences (or strength of preference):

$\Gamma_{3}=\left\{\left(w_{1}, w_{2}, \cdots, w_{n}\right) \in \Gamma_{0} \mid w_{j_{1}}-w_{j_{2}} \geq w_{j_{2}}-w_{j_{3}}\right.$ for all $j_{1} \in \Upsilon_{3}, j_{2} \in \Lambda_{3}$, and $\left.j_{3} \in \Omega_{3}\right\}$,

where $\Upsilon_{3}, \Lambda_{3}$, and $\Omega_{3}$ are three disjoint subsets of $N$.

(iv) An interval bound:

$$
\Gamma_{4}=\left\{\left(w_{1}, w_{2}, \cdots, w_{n}\right) \in \Gamma_{0} \mid \delta_{j_{1}}+\varepsilon_{j_{1}} \geq w_{j_{1}} \geq \delta_{j_{1}} \text { for all } j_{1} \in \Upsilon_{4}\right\},
$$

where $\delta_{j_{1}} \geq 0$ and $\varepsilon_{j_{1}} \geq 0$ are constants that satisfy the condition $0 \leq \delta_{j_{1}} \leq \delta_{j_{1}}+\varepsilon_{j_{1}} \leq 1$, and $\Upsilon_{4}$ is a subset of $N$.

(v) A ratio bound (or a ranking with multiples):

$$
\Gamma_{5}=\left\{\left(w_{1}, w_{2}, \cdots, w_{n}\right) \in \Gamma_{0} \mid w_{j_{1}} \geq \delta_{j_{1} j_{2}} \cdot w_{j_{2}} \text { for all } j_{1} \in \Upsilon_{5} \text { and } j_{2} \in \Lambda_{5}\right\},
$$

where $\delta_{j_{12}}$ is a constant that satisfies the condition $0 \leq \delta_{j_{12}} \leq 1$, and $\Upsilon_{5}$ and $\Lambda_{5}$ are two disjoint subsets of $N$.

The decision maker can provide criteria preferences using one of the five basic ranking forms. Let $\Gamma$ denote a set of the known information about the criterion weights provided by the decision maker, where the known information structure $\Gamma$ consists of the preceding five sets $\Gamma_{1}, \Gamma_{2}, \cdots$, and $\Gamma_{5}$. That is,

$$
\Gamma=\Gamma_{1} \cup \Gamma_{2} \cup \Gamma_{3} \cup \Gamma_{4} \cup \Gamma_{5} .
$$

Considering different types of incomplete preference information, the optimal weight values of the criteria for each alternative $A_{i}$ can be determined via the following linear programming model:

$$
\begin{aligned}
& \text { [M1] } \max \left\{\operatorname{CI}\left(\tilde{A}_{i}\right)=\sum_{j=1}^{n} I\left(\tilde{A}_{i j}\right) \cdot w_{j}\right\} \\
& \text { s.t. } \quad\left(w_{1}, w_{2}, \cdots, w_{n}\right) \in \Gamma,
\end{aligned}
$$

for each $i=1,2, \ldots, m$.

In total, $\mathrm{m}$ linear programming models need to be solved. However, the optimal solutions of [M1] for the m alternatives may be generally different. Thus, a straight-forward comparison between various alternatives is difficult. That is, the corresponding comprehensive inclusion-based indices of all $\mathrm{m}$ alternatives cannot be compared. Because the decision maker cannot easily judge the preference relations among all non-dominated alternatives, we aggregate the $\mathrm{m}$ linear programming models in [M1] as follows:

$$
\begin{aligned}
& \text { [M2] } \max \left\{\sum_{i=1}^{m} C I\left(\tilde{A}_{i}\right)=\sum_{i=1}^{m} \sum_{j=1}^{n} I\left(\tilde{A}_{i j}\right) \cdot w_{j}\right\} \\
& \text { s.t. } \quad\left(w_{1}, w_{2}, \cdots, w_{n}\right) \in \Gamma .
\end{aligned}
$$

The solution results of the linear programming model [M2] yield the optimal weight vector $\bar{w}=\left(\bar{w}_{1}, \bar{w}_{2}, \cdots, \bar{w}_{n}\right)$. The comprehensive inclusion-based index $\overline{C I}\left(\tilde{A}_{i}\right)$ of the characteristics 
$\tilde{A}_{i}$ for alternative $A_{i}$ can then be obtained. The $\mathrm{m}$ alternatives can be ranked according to the decreasing order of $\overline{C I}\left(\tilde{A}_{i}\right)$ for all $A_{i} \in A$. We can then choose the best alternative with the maximum value among all values of $\overline{C I}\left(\tilde{A}_{i}\right)$.

Alternatively, the decision maker might express inconsistent opinions about preferences and criterion importance in the case of contingency. There are no feasible solutions that satisfy all of the conditions in $\Gamma$ for the present circumstances. Therefore, we formulate a multiple-objective, nonlinear programming model by using a goal programming technique to cope with problems related to inconsistent information.

For $j_{1} \neq j_{2} \neq j_{3}$, the conditions in $\Gamma$ are relaxed to $\Gamma^{\prime}$ by introducing several non-negative deviation variables, $e_{(\mathrm{i}) j_{1} j_{2}}^{-}, e_{(\mathrm{ii}) j_{1} j_{2}}^{-}, e_{(\mathrm{iii}) j_{1} j_{2} j_{3}}^{-}, e_{(\mathrm{iv}) j_{1}}^{-}, e_{(\mathrm{iv}) j_{1}}^{+}$, and $e_{(\mathrm{v}) j_{1} j_{2}}^{-}$, which are defined as follows:

$$
\begin{aligned}
\Gamma^{\prime}=\{ & \left(w_{1}, w_{2}, \cdots, w_{n}\right) \in \Gamma_{0} \mid w_{j_{1}}+e_{(\mathrm{i}) j_{1} j_{2}}^{-} \geq w_{j_{2}} \text { for all } j_{1} \in \Upsilon_{1} \text { and } j_{2} \in \Lambda_{1} ; \\
& w_{j_{1}}-w_{j_{2}}+e_{(\mathrm{ii}) j_{1} j_{2}}^{-} \geq \delta_{j_{1} j_{2}} \text { for all } j_{1} \in \Upsilon_{2} \text { and } j_{2} \in \Lambda_{2} ; \\
& w_{j_{1}}-2 w_{j_{2}}+w_{j_{3}}+e_{(\mathrm{iii}) j_{1} j_{2} j_{3}}^{-} \geq 0 \text { for all } j_{1} \in \Upsilon_{3}, j_{2} \in \Lambda_{3}, \text { and } j_{3} \in \Omega_{3} ; \\
& w_{j_{1}}+e_{(\mathrm{iv}) j_{1}}^{-} \geq \delta_{j_{1}}, w_{j_{1}}-e_{(\mathrm{iv}) j_{1}}^{+} \leq \delta_{j_{1}}+\varepsilon_{j_{1}} \text { for all } j_{1} \in \Upsilon_{4} ; \\
& \left.\frac{w_{j_{1}}}{w_{j_{2}}}+e_{(\mathrm{v}) j_{1} j_{2}}^{-} \geq \delta_{j_{1} j_{2}} \text { for all } j_{1} \in \Upsilon_{5} \text { and } j_{2} \in \Lambda_{5}\right\}
\end{aligned}
$$

In the case of inconsistent preference information, the optimal weight values of the criteria can be obtained via the following bi-objective, nonlinear programming model:

$$
\begin{aligned}
& \max \left\{\sum_{i=1}^{m} C I\left(\tilde{A}_{i}\right)=\sum_{i=1}^{m} \sum_{j=1}^{n} I\left(\tilde{A}_{i j}\right) \cdot w_{j}\right\} \\
& \text { [M3] } \min \left\{\sum_{j_{1}, j_{2}, j_{3} \in N}\left(e_{(\mathrm{i}) j_{1} j_{2}}^{-}+e_{(\mathrm{ii}) j_{1} j_{2}}^{-}+e_{(\mathrm{iii}) j_{1} j_{2} j_{3}}^{-}+e_{(\mathrm{iv}) j_{1}}^{-}+e_{(\mathrm{iv}) j_{1}}^{+}+e_{(\mathrm{v}) j_{1} j_{2}}^{-}\right)\right\} \\
& \text {s.t. } \begin{cases}\left(w_{1}, w_{2}, \cdots, w_{n}\right) \in \Gamma^{\prime} & \\
e_{(\mathrm{i}) j_{1} j_{2}}^{-} \geq 0 & j_{1} \in \Upsilon_{1} \text { and } j_{2} \in \Lambda_{1}, \\
e_{(\mathrm{ii}) j_{1} j_{2}}^{-} \geq 0 & j_{1} \in \Upsilon_{2} \text { and } j_{2} \in \Lambda_{2}, \\
e_{(\mathrm{iii}) j_{1} j_{2} j_{3}}^{-} \geq 0 & j_{1} \in \Upsilon_{3}, j_{2} \in \Lambda_{3}, \text { and } j_{3} \in \Omega_{3}, \\
e_{(\mathrm{iv}) j_{1}}^{-} \geq 0, e_{(\mathrm{iv}) j_{1}}^{+} \geq 0 & j_{1} \in \Upsilon_{4}, \\
e_{(\mathrm{v}) j_{1} j_{2}}^{-} \geq 0 & j_{1} \in \Upsilon_{5} \text { and } j_{2} \in \Lambda_{5} .\end{cases}
\end{aligned}
$$

The second objective function in [M3] is equivalent to the following objective function: $\max \left\{-\sum\left(e_{(\mathrm{i}) j_{1} j_{2}}^{-}+e_{(\mathrm{ii}) j_{1} j_{2}}^{-}+e_{(\mathrm{iii}) j_{1} j_{2} j_{3}}^{-}+e_{(\mathrm{iv}) j_{1}}^{-}+e_{(\mathrm{iv}) j_{1}}^{+}+e_{(\mathrm{v}) j_{1} j_{2}}^{-}\right)\right\}$. By utilizing the max-min operator, the model in [M3] can be integrated into the following single-objective nonlinear programming model: 
$\max \lambda$

$$
\text { s.t. } \begin{cases}\sum_{i=1}^{m} \sum_{j=1}^{n} I\left(\tilde{A}_{i j}\right) \cdot w_{j} \geq \lambda, \\ -\sum_{j_{1}, j_{2}, j_{3} \in N}\left(e_{(\mathrm{i}) j_{1} j_{2}}^{-}+e_{(\mathrm{ii}) j_{1} j_{2}}^{-}+e_{(\mathrm{iii}) j_{1} j_{2} j_{3}}^{-}+e_{(\mathrm{iv}) j_{1}}^{-}+e_{(\mathrm{iv}) j_{1}}^{+}+e_{(\mathrm{v}) j_{1} j_{2}}^{-}\right) \geq \lambda, \\ \left(w_{1}, w_{2}, \cdots, w_{n}\right) \in \Gamma^{\prime}, & \\ e_{(\mathrm{i}) j_{1} j_{2}}^{-} \geq 0 & j_{1} \in \Upsilon_{1} \text { and } j_{2} \in \Lambda_{1}, \\ e_{(\mathrm{ii}) j_{1} j_{2}}^{-} \geq 0 & j_{1} \in \Upsilon_{2} \text { and } j_{2} \in \Lambda_{2}, \\ e_{(\mathrm{iii}) j_{1} j_{2} j_{3}}^{-} \geq 0 & j_{1} \in \Upsilon_{3}, j_{2} \in \Lambda_{3}, \text { and } j_{3} \in \Omega_{3}, \\ e_{(\mathrm{iv}) j_{1}}^{-} \geq 0, e_{(\mathrm{iv}) j_{1}}^{+} \geq 0 & j_{1} \in \Upsilon_{4}, \\ e_{(\mathrm{v}) j_{1} j_{2}}^{-} \geq 0 & j_{1} \in \Upsilon_{5} \text { and } j_{2} \in \Lambda_{5} .\end{cases}
$$

Solving the integrated nonlinear programming model [M4] yields the optimal weight vector $\overline{\bar{w}}=\left(\overline{\bar{w}}_{1}, \overline{\bar{w}}_{2}, \cdots, \overline{\bar{w}}_{n}\right)$ and the optimal deviation values $\overline{\bar{e}}_{(\mathrm{i}) j_{1} j_{2}}, \overline{\bar{e}}_{(\mathrm{ii}) j_{1} j_{2}}, \overline{\bar{e}}_{(\mathrm{iii}) j_{1} j_{2} j_{3}}, \overline{\bar{e}}_{(\overline{\mathrm{iv}}) j_{1}}$, $\overline{\bar{e}}_{(\mathrm{iv}) j_{1}}^{+}$, and $\overline{\bar{e}}_{(\mathrm{v}) j_{1} j_{2}}^{-}\left(j_{1}, j_{2}, j_{3} \in N\right)$. Next, the corresponding comprehensive inclusion-based index $\overline{\overline{C I}}\left(\tilde{A}_{i}\right)$ of the characteristics $\tilde{A}_{i}$ for the alternative $A_{i}$ can also be obtained. The final step is to choose the maximum among all values of $\overline{\overline{C I}}\left(\tilde{A}_{i}\right)$ and obtain the optimal ranking of the alternatives according to the decreasing order of $\overline{\overline{C I}}\left(\tilde{A}_{i}\right)$ for all $A_{i} \in A$.

\subsection{The proposed algorithm}

Based on the concept of inclusion comparison possibilities, the proposed interval-valued intuitionistic fuzzy MCDA method with comprehensive inclusion-based indexing under incomplete preference information can be summarized in the following steps:

Step 1: Formulate a multiple criteria decision-making problem. Specify the alternative set $A=\left\{A_{1}, A_{2}, \cdots, A_{m}\right\}$ and the criterion set $X=\left\{x_{1}, x_{2}, \cdots, x_{n}\right\}$, which is divided into $X_{b}$ and $X_{c}$.

Step 2: Determine the evaluation $\tilde{A}_{i j}$ for every $A_{i} \in A$ and $x_{j} \in X$ to establish the interval-valued intuitionistic fuzzy decision matrix $\tilde{D}$.

Step 3: Collect preference information from the decision maker as criterion weights, in terms of the weak order, strict order, difference order, interval bound, or ratio bound. Construct the set $\Gamma$ of the known information.

Step 4: Identify the interval-valued intuitionistic fuzzy positive-ideal and negative-ideal solutions $\tilde{A}_{\star}$ and $\tilde{A}_{-}$, respectively, and the corresponding evaluations $\tilde{A}_{\star_{j}}$ with respect to each $x_{j}$.

Step 5: Compute the inclusion comparison possibilities $\underset{\tilde{\tilde{A}}}{p}\left(\tilde{A}_{\star_{j}} \supseteq \tilde{A}_{i j}\right), p\left(\tilde{A}_{i j} \supseteq \tilde{A}_{-j}\right)$ (for every $A_{i} \in A$ and $\left.x_{j} \in X_{b}\right), p\left(\tilde{A}_{i j} \supseteq \tilde{A}_{\star_{j}}\right)$, and $p\left(\tilde{A}_{-j} \supseteq \tilde{A}_{i j}\right)$ (for every $A_{i} \in A$ and $\left.x_{j} \in X_{c}\right)$. 
Step 6: Calculate the inclusion-based index $I\left(\tilde{A}_{i j}\right)$ of $\tilde{A}_{i j}$. Then, given the weight vector $\left(w_{1}, w_{2}, \cdots, w_{n}\right)$, determine the comprehensive inclusion-based index $\operatorname{CI}\left(\tilde{A}_{i}\right)$ for the characteristics $\tilde{A}_{i}$.

Step 7: Construct the linear programming model [M2] (with consistent weight information) or the integrated nonlinear programming model [M4] (with inconsistent weight information).

Step 8: Solve [M2] (or [M4]) to acquire the optimal weight vector $\bar{w}$ (or $\overline{\bar{w}}$ ) and the comprehensive inclusion-based index $\overline{C I}\left(\tilde{A}_{i}\right)$ (or $\overline{\overline{C I}}\left(\tilde{A}_{i}\right)$ ) of $\tilde{A}_{i}$. Rank the m alternatives in accordance with the $\overline{C I}\left(\tilde{A}_{i}\right)$ (or $\left.\overline{\overline{C I}}\left(\tilde{A}_{i}\right)\right)$ values. The alternative with the largest $\overline{C I}\left(\tilde{A}_{i}\right)$ (or $\left.\overline{\overline{C I}}\left(\tilde{A}_{i}\right)\right)$ value is the best choice.

\section{Case illustration and a comparative study}

In this section, a realistic example involving an MCDA problem, i.e., selecting a suitable bridge construction method, is used as an illustration of applying the proposed interval-valued intuitionistic fuzzy MCDA method using inclusion comparison possibilities under incomplete preference information.

\subsection{Problem description}

Some of the most famous scenic spots in Taiwan are the Hualian and Taidong areas (hereafter referred to as Huadong). The Su-Hua Highway is a vital route from the Taipei metropolitan area to Huadong. Because of the winding nature of the Su-Hua Highway, accidents frequently occur. Furthermore, poor weather conditions and strong, windy storms (such as typhoons) frequently cause serious disasters that may result in collapsed roads or restricted access. To address these conditions, the government formulated the Su-Hua Highway Initiative; unfortunately, the plan did not pass a 2008 environmental assessment and had to be suspended. In response, the government launched a new initiative, the Su-Hua Highway Alternative Route Plan, to present a safe and high-speed alternative to the most dangerous segment of the Su-Hua Highway. This plan increases the convenience of travel from Taipei to Huadong and promotes economic development in the Huadong area. However, experts and scholars are concerned that the plan could destroy the integrity of the original ecology and environment.

This study explores the question of how to select the most suitable bridge construction method for the Su-Hua Highway Alternative Route Plan. This study considers the four most commonly used bridge construction methods:

1. Advanced shoring method $\left(\mathrm{A}_{1}\right)$ - to minimize environmental pollution and other effects, this method maintains the original ecological and environmental conditions. Additionally, the suitability of this method for the proposed site is very high. This method will not be adversely affected by the topographical conditions. However, the damage costs associated with this method are relatively high, and the durability of the bridge is low. 
2. Incremental launching method $\left(A_{2}\right)$ - this method lowers construction costs through the use of reusable materials. Moreover, this method has a low degree of disruption to daily traffic patterns. However, this method has many negative effects on the surrounding scenery.

3. Balanced cantilever method $\left(A_{3}\right)$ - under this method, quality control is very effective; therefore, the lifespan of the bridge is long, and the frequency of necessary maintenance and repair is low. However, the initial costs incurred under this method are high, and weather conditions could delay construction and affect the schedule.

4. Precast segmental method $\left(A_{4}\right)$ - this method also has little effect on existing traffic patterns during the construction period and has little effect on the landscape. However, this method requires increased attention to the climate patterns and the location of the project because the method has many limitations regarding the construction site and geography.

Several criteria are considered for evaluating bridge construction methods and for serving as bases with which the proper construction method can be selected. These criteria are as follows:

1. Durability $\left(x_{1}\right)$ - the number of years of use, the long-term use outlook, and the avoidance of frequent maintenance.

2. Damage cost $\left(x_{2}\right)$ - the cost of damage or maintenance of the bridge.

3. Construction cost $\left(x_{3}\right)$ - the expenses incurred from the design phase to the completion of construction.

4. Traffic conflict $\left(x_{4}\right)$ - the degree to which construction negatively affects normal traffic patterns during the period of construction.

5. Site condition $\left(x_{5}\right)$ - the condition of the location, including the configuration of the topography, the suitability of the construction method for the site, and whether the location imposes restrictions on a chosen construction method.

6. Weather condition $\left(x_{6}\right)$ - a measure of whether climate accelerates bridge damage or the possibility that weather conditions could cause construction delays.

7. Landscape $\left(x_{7}\right)$ - whether the construction method has harmful effects on the scenic nature of the neighboring or surrounding areas and whether the appearance of the bridge negatively affects the surrounding landscape.

8. Environmental effect $\left(x_{8}\right)$ - the degree of environmental damage or negative effects on the surrounding areas during construction and use.

\subsection{Illustration of the algorithm}

This application explores the problem of how to select the most suitable bridge construction method for the Su-Hua Highway Alternative Route Plan at the Hualian and Taidong areas in Taiwan. The proposed interval-valued intuitionistic fuzzy MCDA method was applied to solve this problem, and the computational procedure is summarized as follows:

Step 1: The authority considers the four most commonly used bridge construction methods: the advanced shoring method $\left(A_{1}\right)$, incremental launching method $\left(A_{2}\right)$, balanced 
cantilever method $\left(A_{3}\right)$, and precast segmental method $\left(A_{4}\right)$. The set of candidate methods is denoted by $A=\left\{A_{1}, A_{2}, A_{3}, A_{4}\right\}$. Eight criteria for evaluating the bridge construction methods are selected, including durability $\left(x_{1}\right)$, damage cost $\left(x_{2}\right)$, construction cost $\left(x_{3}\right)$, traffic conflict $\left(x_{4}\right)$, site condition $\left(x_{5}\right)$, weather condition $\left(x_{6}\right)$, landscape $\left(x_{7}\right)$, and environmental effect $\left(x_{8}\right)$. The set of evaluative criteria is denoted by $X=\left\{x_{1}, x_{2}, \cdots, x_{8}\right\}$, with $X_{b}=\left\{x_{1}, x_{5}\right\}$ and $X_{c}=\left\{x_{2}, x_{3}, x_{4}, x_{6}, x_{7}, x_{8}\right\}$.

Step 2: The four bridge construction methods were evaluated based on the eight criteria. The evaluation $\tilde{A}_{i j}$ of alternative $A_{i} \in A(i=1,2,3,4)$ on $x_{j} \in X \quad(\mathrm{j}=1,2, \ldots, 8)$ are presented in Table 1 . Then, we construct the interval-valued intuitionistic fuzzy decision matrix $\tilde{D}$.

Table 1. The evaluation $\tilde{A}_{i j}$ in the decision matrix $\tilde{D}$

\begin{tabular}{clll}
\hline$\tilde{A}_{11}$ & $([0.28,0.35],[0.33,0.46])$ & $\tilde{A}_{31}$ & $([0.72,0.77],[0.17,0.20])$ \\
\hline$\tilde{A}_{12}$ & $([0.43,0.58],[0.16,0.17])$ & $\tilde{A}_{32}$ & $([0.03,0.07],[0.66,0.76])$ \\
\hline$\tilde{A}_{13}$ & $([0.08,0.16],[0.63,0.75])$ & $\tilde{A}_{33}$ & $([0.05,0.18],[0.36,0.63])$ \\
\hline$\tilde{A}_{14}$ & $([0.07,0.49],[0.38,0.41])$ & $\tilde{A}_{34}$ & $([0.35,0.45],[0.39,0.44])$ \\
\hline$\tilde{A}_{15}$ & $([0.64,0.67],[0.15,0.33])$ & $\tilde{A}_{35}$ & $([0.64,0.67],[0.15,0.33])$ \\
\hline$\tilde{A}_{16}$ & $([0.07,0.14],[0.64,0.74])$ & $\tilde{A}_{36}$ & $([0.14,0.36],[0.22,0.40])$ \\
\hline$\tilde{A}_{17}$ & $([0.14,0.21],[0.34,0.37])$ & $\tilde{A}_{37}$ & $([0.18,0.19],[0.68,0.74])$ \\
\hline$\tilde{A}_{18}$ & $([0.04,0.09],[0.88,0.90])$ & $\tilde{A}_{38}$ & $([0.36,0.40],[0.44,0.58])$ \\
\hline$\tilde{A}_{21}$ & $([0.68,0.71],[0.06,0.26])$ & $\tilde{A}_{41}$ & $([0.37,0.52],[0.33,0.41])$ \\
\hline$\tilde{A}_{22}$ & $([0.04,0.12],[0.61,0.86])$ & $\tilde{A}_{42}$ & $([0.26,0.36],[0.46,0.64])$ \\
\hline$\tilde{A}_{23}$ & $([0.09,0.26],[0.33,0.46])$ & $\tilde{A}_{43}$ & $([0.36,0.40],[0.52,0.52])$ \\
\hline$\tilde{A}_{24}$ & $([0.12,0.23],[0.64,0.67])$ & $\tilde{A}_{44}$ & $([0.17,0.40],[0.29,0.48])$ \\
\hline$\tilde{A}_{25}$ & $([0.37,0.39],[0.26,0.29])$ & $\tilde{A}_{45}$ & $([0.15,0.31],[0.21,0.62])$ \\
\hline$\tilde{A}_{26}$ & $([0.18,0.19],[0.74,0.78])$ & $\tilde{A}_{46}$ & $([0.26,0.85],[0.13,0.14])$ \\
\hline$\tilde{A}_{27}$ & $([0.49,0.66],[0.18,0.26])$ & $\tilde{A}_{47}$ & $([0.04,0.15],[0.83,0.84])$ \\
\hline$\tilde{A}_{28}$ & $([0.18,0.41],[0.17,0.28])$ & $\tilde{A}_{48}$ & $([0.13,0.21],[0.75,0.77])$ \\
\hline & & & \\
\hline & & & \\
\hline & & & \\
\hline
\end{tabular}

Step 3: The authority was provided with the preference for all criteria, and the known information about criterion weights is indicated as follows: 


$$
\begin{gathered}
\Gamma_{1}=\left\{\left(w_{1}, w_{2}, \cdots, w_{8}\right) \in \Gamma_{0} \mid w_{5} \geq w_{6}\right\}, \\
\Gamma_{2}=\left\{\left(w_{1}, w_{2}, \cdots, w_{8}\right) \in \Gamma_{0} \mid 0.12 \geq w_{8}-w_{2} \geq 0.07\right\}, \\
\Gamma_{3}=\left\{\left(w_{1}, w_{2}, \cdots, w_{8}\right) \in \Gamma_{0} \mid w_{7}-w_{2} \geq w_{2}-w_{4}\right\}, \\
\Gamma_{4}=\left\{\left(w_{1}, w_{2}, \cdots, w_{8}\right) \in \Gamma_{0} \mid 0.20 \geq w_{1} \geq 0.15,0.16 \geq w_{5} \geq 0.11\right\}, \\
\Gamma_{5}=\left\{\left(w_{1}, w_{2}, \cdots, w_{8}\right) \in \Gamma_{0} \mid w_{3} \geq 0.6 \cdot w_{5}\right\},
\end{gathered}
$$

where $\Gamma_{0}=\left\{\left(w_{1}, w_{2}, \cdots, w_{8}\right) \mid w_{j} \geq 0(j=1,2, \cdots, 8), \sum_{j=1}^{8} w_{j}=1\right\}$. It follows that the set $\Gamma$ of the known information about criterion weights is as follows:

$$
\begin{aligned}
& \Gamma=\left\{\left(w_{1}, w_{2}, \cdots, w_{8}\right) \in \Gamma_{0} \mid w_{5} \geq w_{6}, 0.12 \geq w_{8}-w_{2} \geq 0.07, w_{7}-w_{2} \geq w_{2}-w_{4}, 0.20 \geq w_{1} \geq\right. \\
&\left.0.15,0.16 \geq w_{5} \geq 0.11, w_{3} \geq 0.6 \cdot w_{5}\right\} .
\end{aligned}
$$

Step 4: The interval-valued intuitionistic fuzzy positive-ideal solution $\tilde{A}_{\star}$ was identified using (16) and (17):

$$
\begin{aligned}
\tilde{A}_{\star}= & \left\{\left\langle x_{1},\left(\mu_{\star_{1}}, v_{\star_{1}}\right)\right\rangle,\left\langle x_{2},\left(\mu_{\star_{2}}, v_{\star_{2}}\right)\right\rangle, \cdots,\left\langle x_{8},\left(\mu_{\star_{8}}, v_{\star_{8}}\right)\right\rangle\right\}= \\
& \left\{\left\langle x_{1},([0.72,0.77],[0.06,0.20])\right\rangle,\left\langle x_{2},([0.03,0.07],[0.66,0.86])\right\rangle,\right. \\
& \left\langle x_{3},([0.05,0.16],[0.63,0.75])\right\rangle,\left\langle x_{4},([0.07,0.23],[0.64,0.67])\right\rangle, \\
& \left\langle x_{5},([0.64,0.67],[0.15,0.29])\right\rangle,\left\langle x_{6},([0.07,0.14],[0.74,0.78])\right\rangle, \\
& \left.\left\langle x_{7},([0.04,0.15],[0.83,0.84])\right\rangle,\left\langle x_{8},([0.04,0.09],[0.88,0.90])\right\rangle\right\} .
\end{aligned}
$$

Applying (20) and (21), the interval-valued intuitionistic fuzzy negative-ideal solution $\tilde{A}_{-}$is given by:

$$
\begin{aligned}
\tilde{A}_{-}= & \left\{\left\langle x_{1},\left(\mu_{-1}, v_{-1}\right)\right\rangle,\left\langle x_{2},\left(\mu_{-2}, v_{-2}\right)\right\rangle, \cdots,\left\langle x_{8},\left(\mu_{-8}, v_{-8}\right)\right\rangle\right\}= \\
& \left\{\left\langle x_{1},([0.28,0.35],[0.33,0.46])\right\rangle,\left\langle x_{2},([0.43,0.58],[0.16,0.17])\right\rangle,\right. \\
& \left\langle x_{3},([0.36,0.40],[0.33,0.46])\right\rangle,\left\langle x_{4},([0.35,0.49],[0.29,0.41])\right\rangle, \\
& \left\langle x_{5},([0.15,0.31],[0.26,0.62])\right\rangle,\left\langle x_{6},([0.26,0.85],[0.13,0.14])\right\rangle, \\
& \left.\left\langle x_{7},([0.49,0.66],[0.18,0.26])\right\rangle,\left\langle x_{8},([0.36,0.41],[0.17,0.28])\right\rangle\right\} .
\end{aligned}
$$

Step 5: We calculated the required inclusion comparison possibilities. Consider $p\left(\tilde{A}_{{ }_{5}} \supseteq \tilde{A}_{25}\right)$ for example. Applying (11) and (12), we have:

$$
\begin{aligned}
p^{-}\left(\tilde{A}_{\star_{5}} \supseteq \tilde{A}_{25}\right)=\max \left\{1-\max \left\{\frac{\left(1-v_{25}^{-}\right)-\mu_{\star_{5}}^{-}}{\left(1-\mu_{\star_{5}}^{-}-v_{\star_{5}}^{+}\right)+\left(1-\mu_{25}^{+}-v_{25}^{-}\right)}, 0\right\}, 0\right\}= \\
\max \left\{1-\max \left\{\frac{(1-0.26)-0.64}{(1-0.64-0.29)+(1-0.39-0.26)}, 0\right\}, 0\right\}=0.7619,
\end{aligned}
$$




$$
\begin{aligned}
p^{+}\left(\tilde{A}_{\star_{5}} \supseteq \tilde{A}_{25}\right)= & \max \left\{1-\max \left\{\frac{\left(1-v_{25}^{+}\right)-\mu_{\star_{5}}^{+}}{\left(1-\mu_{\star_{5}}^{+}-v_{\star_{5}}^{-}\right)+\left(1-\mu_{25}^{-}-v_{25}^{+}\right)}, 0\right\}, 0\right\}= \\
& \max \left\{1-\max \left\{\frac{(1-0.29)-0.67}{(1-0.67-0.15)+(1-0.37-0.29)}, 0\right\}, 0\right\}=0.9231 .
\end{aligned}
$$

The inclusion comparison probability $p\left(\tilde{A}_{{ }_{5}} \supseteq \tilde{A}_{25}\right)$ using (13) is computed as follows: $p\left(\tilde{A}_{*_{5}} \supseteq \tilde{A}_{25}\right)=\frac{1}{2}\left(p^{-}\left(\tilde{A}_{*_{5}} \supseteq \tilde{A}_{25}\right)+p^{+}\left(\tilde{A}_{*_{5}} \supseteq \tilde{A}_{25}\right)\right)=0.8425$. For every $A_{i} \in A$, we calculated $p\left(\tilde{A}_{{ }_{j}} \supseteq \tilde{A}_{i j}\right)$ and $p\left(\tilde{A}_{i j} \supseteq \tilde{A}_{-j}\right)$ for the benefit criteria and $p\left(\tilde{A}_{i j} \supseteq \tilde{A}_{{ }_{j}}\right)$ and $p\left(\tilde{A}_{-j} \supseteq \tilde{A}_{i j}\right)$ for the cost criteria. These computation results are indicated in Table 2.

\begin{tabular}{|c|c|c|c|c|c|c|c|c|c|}
\hline & $A_{1}$ & $A_{2}$ & $A_{3}$ & $A_{4}$ & & $A_{1}$ & $A_{2}$ & $A_{3}$ & $A_{4}$ \\
\hline$p\left(\tilde{A}_{\star_{1}} \supseteq \tilde{A}_{i 1}\right)$ & 1.0000 & 0.6452 & 0.5471 & 1.0000 & $p\left(\tilde{A}_{i 1} \supseteq \tilde{A}_{-1}\right)$ & 0.5000 & 1.0000 & 1.0000 & 0.6978 \\
\hline$p\left(\tilde{A}_{i 2} \supseteq \tilde{A}_{\star_{2}}\right)$ & 0.8302 & 1.0000 & 1.0000 & 1.0000 & $p\left(\tilde{A}_{-2} \supseteq \tilde{A}_{i 2}\right)$ & 0.9125 & 0.2170 & 0.2272 & 0.6218 \\
\hline$p\left(\tilde{A}_{i 3} \supseteq \tilde{A}_{*_{3}}\right)$ & 1.0000 & 0.9677 & 0.9925 & 1.0000 & $p\left(\tilde{A}_{-3} \supseteq \tilde{A}_{i 3}\right)$ & 0.0417 & 0.3135 & 0.2123 & 0.2476 \\
\hline$p\left(\tilde{A}_{i 4} \supseteq \tilde{A}_{*_{4}}\right)$ & 1.0000 & 1.0000 & 1.0000 & 0.9205 & $p\left(\tilde{A}_{-4} \supseteq \tilde{A}_{i 4}\right)$ & 0.5898 & 0.1000 & 0.5877 & 0.4894 \\
\hline$p\left(\tilde{A}_{\star_{5}} \supseteq \tilde{A}_{i 5}\right)$ & 0.5800 & 0.8425 & 0.5800 & 0.8636 & $p\left(\tilde{A}_{i 5} \supseteq \tilde{A}_{-5}\right)$ & 0.8913 & 0.7597 & 0.8913 & 0.5037 \\
\hline$p\left(\tilde{A}_{i 6} \supseteq \tilde{A}_{* 6}\right)$ & 1.0000 & 1.0000 & 0.9630 & 0.4824 & $p\left(\tilde{A}_{-6} \supseteq \tilde{A}_{i 6}\right)$ & 0.5551 & 0.5625 & 0.7170 & 0.8000 \\
\hline$p\left(\tilde{A}_{i 7} \supseteq \tilde{A}_{{ }^{\prime} 7}\right)$ & 1.0000 & 1.0000 & 1.0000 & 1.0000 & $p\left(\tilde{A}_{-7} \supseteq \tilde{A}_{i 7}\right)$ & 0.6434 & 0.9800 & 0.2414 & 0.0000 \\
\hline$p\left(\tilde{A}_{i 8} \supseteq \tilde{A}_{* 8}\right)$ & 1.0000 & 1.0000 & 1.0000 & 1.0000 & $p\left(\tilde{A}_{-8} \supseteq \tilde{A}_{i 8}\right)$ & 0.0000 & 0.6373 & 0.5029 & 0.0870 \\
\hline
\end{tabular}

Table 2. The results of the inclusion comparison possibilities

Step 6: We calculated the inclusion-based index $I\left(\tilde{A}_{i j}\right)$ for every $A_{i} \in A$ and $x_{j} \in X$; the computation results are listed in Table 3. Consider $I\left(\tilde{A}_{31}\right)$ and $I\left(\tilde{A}_{36}\right)$ for example. Applying (23), we have:

$$
\begin{gathered}
I\left(\tilde{A}_{31}\right)=\frac{p\left(\tilde{A}_{31} \supseteq \tilde{A}_{-1}\right)}{p\left(\tilde{A}_{{ }_{1}} \supseteq \tilde{A}_{31}\right)+p\left(\tilde{A}_{31} \supseteq \tilde{A}_{-1}\right)}=\frac{1.0000}{0.5471+1.0000}=0.6464, \\
I\left(\tilde{A}_{36}\right)=\frac{p\left(\tilde{A}_{-6} \supseteq \tilde{A}_{36}\right)}{p\left(\tilde{A}_{36} \supseteq \tilde{A}_{* 6}\right)+p\left(\tilde{A}_{-6} \supseteq \tilde{A}_{36}\right)}=\frac{0.7170}{0.9630+0.7170}=0.4268 .
\end{gathered}
$$


Table 3. The results of the inclusion-based indices

\begin{tabular}{cccccccccc}
\hline & $A_{1}$ & $A_{2}$ & $A_{3}$ & $A_{4}$ & & $A_{1}$ & $A_{2}$ & $A_{3}$ & $A_{4}$ \\
\hline$I\left(\tilde{A}_{i 1}\right)$ & 0.3333 & 0.6078 & 0.6464 & 0.4110 & $I\left(\tilde{A}_{i 5}\right)$ & 0.6058 & 0.4742 & 0.6058 & 0.3684 \\
\hline$I\left(\tilde{A}_{i 2}\right)$ & 0.5236 & 0.1783 & 0.1851 & 0.3834 & $I\left(\tilde{A}_{i 6}\right)$ & 0.3570 & 0.3600 & 0.4268 & 0.6238 \\
\hline$I\left(\tilde{A}_{i 3}\right)$ & 0.0400 & 0.2447 & 0.1762 & 0.1985 & $I\left(\tilde{A}_{i 7}\right)$ & 0.3915 & 0.4949 & 0.1945 & 0.0000 \\
\hline$I\left(\tilde{A}_{i 4}\right)$ & 0.3710 & 0.0909 & 0.3702 & 0.3471 & $I\left(\tilde{A}_{i 8}\right)$ & 0.0000 & 0.3892 & 0.3346 & 0.0800 \\
\hline
\end{tabular}

Applying (25), the comprehensive inclusion-based indices for all $\tilde{A}_{i}$ are as follows:

$$
\begin{aligned}
C I\left(\tilde{A}_{1}\right)= & 0.3333 w_{1}+0.5236 w_{2}+0.0400 w_{3}+0.3710 w_{4}+0.6058 w_{5}+0.3570 w_{6}+ \\
& 0.3915 w_{7}+0.0000 w_{8}, \\
C I\left(\tilde{A}_{2}\right)= & 0.6078 w_{1}+0.1783 w_{2}+0.2447 w_{3}+0.0909 w_{4}+0.4742 w_{5}+0.3600 w_{6}+ \\
& 0.4949 w_{7}+0.3892 w_{8}, \\
C I\left(\tilde{A}_{3}\right)= & 0.6464 w_{1}+0.1851 w_{2}+0.1762 w_{3}+0.3702 w_{4}+0.6058 w_{5}+0.4268 w_{6}+ \\
& 0.1945 w_{7}+0.3346 w_{8}, \\
C I\left(\tilde{A}_{4}\right)= & 0.4110 w_{1}+0.3834 w_{2}+0.1985 w_{3}+0.3471 w_{4}+0.3684 w_{5}+0.6238 w_{6}+ \\
& 0.0000 w_{7}+0.0800 w_{8} .
\end{aligned}
$$

Step 7: Because there is no inconsistent weight information in the set $\Gamma$, we applied [M2] to construct the following linear programming model:

$$
\begin{gathered}
\max \left\{C I\left(\tilde{A}_{1}\right)+C I\left(\tilde{A}_{2}\right)+C I\left(\tilde{A}_{3}\right)+C I\left(\tilde{A}_{4}\right)=1.9985 w_{1}+1.2705 w_{2}+0.6594 w_{3}+\right. \\
\left.1.1792 w_{4}+2.0541 w_{5}+1.7676 w_{6}+1.0809 w_{7}+0.8039 w_{8}\right\}
\end{gathered}
$$

subject to

$$
\begin{aligned}
& w_{5} \geq w_{6}, 0.12 \geq w_{8}-w_{2} \geq 0.07, w_{7}-w_{2} \geq w_{2}-w_{4}, 0.20 \geq w_{1} \geq 0.15,0.16 \geq w_{5} \geq \\
& 0.11, w_{3} \geq 0.6 \cdot w_{5}, w_{1}+w_{2}+w_{3}+w_{4}+w_{5}+w_{6}+w_{7}+w_{8}=1, w_{j} \geq 0 \text { for all } j .
\end{aligned}
$$

Step 8: Solving the above linear programming model, the optimal objective value is 1.5010 and the optimal weight vector $\bar{w}=(0.2,0,0.096,0.314,0.16,0.16,0,0.07)$. The obtained results of the comprehensive inclusion-based indices are given by: $=0.3410, \overline{C I}\left(\tilde{A}_{2}\right)=$ $0.3343, \overline{C I}\left(\tilde{A}_{3}\right)=0.4511$, and $\overline{C I}\left(\tilde{A}_{4}\right)=0.3746$. The ranking order of the candidate bridge construction methods is $A_{3} \succ A_{4} \succ A_{1} \succ A_{2}$. Therefore, the best choice is the balanced cantilever method $\left(A_{3}\right)$. 


\subsection{Remarks on the case of inconsistent weight information}

Consider the same bridge construction selection problem with inconsistent weight information. Assume that we add the condition $0.05 \geq w_{6}-w_{5} \geq 0.01$ to the set $\Gamma_{2}$ in Step 3. The sets $\Gamma_{2}$ and $\mathrm{G}$ are updated as follows:

$$
\begin{gathered}
\Gamma_{2}^{(\text {new })}=\left\{\left(w_{1}, w_{2}, \cdots, w_{8}\right) \in \Gamma_{0} \mid 0.12 \geq w_{8}-w_{2} \geq 0.07,0.05 \geq w_{6}-w_{5} \geq 0.01\right\}, \\
\Gamma^{(\text {new })}=\left\{\left(w_{1}, w_{2}, \cdots, w_{8}\right) \in \Gamma_{0} \mid w_{5} \geq w_{6}, 0.12 \geq w_{8}-w_{2} \geq 0.07,0.05 \geq w_{6}-w_{5} \geq 0.01,\right. \\
\left.w_{7}-w_{2} \geq w_{2}-w_{4}, 0.20 \geq w_{1} \geq 0.15,0.16 \geq w_{5} \geq 0.11, w_{3} \geq 0.6 \cdot w_{5}\right\} .
\end{gathered}
$$

The conditions of $w_{5} \geq w_{6}$ in $\Gamma_{1}$ and $0.05 \geq w_{6}-w_{5} \geq 0.01$ in $\Gamma_{2}^{(\text {new })}$ are conflicting, and thus, the weight information in $\Gamma^{\text {(new) }}$ is partially inconsistent. Because inconsistent weight information exists in $\Gamma^{(\mathrm{new})}$, we applied [M4] in Step 7 to construct the following integrated nonlinear programming model:

$\max \lambda$

subject to

$$
\begin{aligned}
& 1.9985 w_{1}+1.2705 w_{2}+0.6594 w_{3}+1.1792 w_{4}+2.0541 w_{5}+1.7676 w_{6}+1.0809 w_{7}+ \\
& 0.8039 w_{8} \geq \lambda, \\
& -\left(e_{(\mathrm{i}) 56}^{-}+e_{(\mathrm{ii}) 82}^{-}+e_{(\mathrm{ii}) 65}^{-}+e_{(\mathrm{ii}) 82}^{+}+e_{(\mathrm{ii}) 65}^{+}+e_{(\mathrm{iii}) 724}^{-}+e_{(\mathrm{iv}) 1}^{-}+e_{(\mathrm{iv}) 5}^{-}+e_{(\mathrm{iv}) 1}^{+}+e_{(\mathrm{iv}) 5}^{+}+e_{(\mathrm{v}) 35}^{-}\right) \geq \lambda, \\
& w_{5}+e_{(\mathrm{i}) 56}^{-} \geq w_{6}, w_{8}-w_{2}+e_{(\mathrm{ii}) 82}^{-} \geq 0.07, w_{6}-w_{5}+e_{(\mathrm{ii}) 65}^{-} \geq 0.01, w_{8}-w_{2}-e_{(\mathrm{ii}) 82}^{+} \leq 0.12, \\
& w_{6}-w_{5}-e_{(\mathrm{ii}) 65}^{+} \leq 0.05, w_{7}-2 w_{2}+w_{4}+e_{(\mathrm{iii}) 724}^{-} \geq 0, w_{1}+e_{(\mathrm{iv}) 1}^{-} \geq 0.15, w_{5}+e_{(\mathrm{iv}) 5}^{-} \geq 0.11, \\
& w_{1}-e_{(\mathrm{iv}) 1}^{+} \leq 0.20, w_{5}-e_{(\mathrm{iv}) 5}^{+} \leq 0.16, \frac{w_{3}}{w_{5}}+e_{(\mathrm{v}) 35}^{-} \geq 0.6, \\
& w_{1}+w_{2}+w_{3}+w_{4}+w_{5}+w_{6}+w_{7}+w_{8}=1, w_{j} \geq 0 \text { for all } j, \\
& e_{(\mathrm{i}) 56}^{-}, e_{(\mathrm{ii}) 82}^{-}, e_{(\mathrm{ii}) 65}^{-}, e_{(\mathrm{ii}) 82}^{+}, e_{(\mathrm{ii}) 65}^{+}, e_{(\mathrm{iii}) 724}^{-}, e_{(\mathrm{iv}) 1}^{-}, e_{(\mathrm{iv}) 5}^{-}, e_{(\mathrm{iv}) 1}^{+}, e_{(\mathrm{iv}) 5}^{+}, e_{(\mathrm{v}) 35}^{-} \geq 0,
\end{aligned}
$$

where $e_{(\mathrm{i}) 56}^{-}, e_{(\mathrm{ii}) 82}^{-}, e_{(\mathrm{ii}) 65}^{-}, e_{(\mathrm{ii}) 82}^{+}, e_{(\mathrm{ii}) 65}^{+}, e_{(\mathrm{iii}) 724}^{-}, e_{(\mathrm{iv}) 1}^{-}, e_{(\mathrm{iv}) 5}^{-}, e_{(\mathrm{iv}) 1}^{+}, e_{(\mathrm{iv}) 5}^{+}$, and $e_{(\mathrm{v}) 35}^{-}$ are deviation variables.

The above nonlinear programming model was solved by Step 8 to obtain the optimal objective value, $\overline{\bar{\lambda}}=-0.01$, the optimal weight vector, $\overline{\bar{w}}=(0.15,0,0.56,0,0.11,0.11,0,0.07)$, the optimal deviation values, $\overline{\bar{e}}_{(\mathrm{ii}) 65}=0.01$, and $\overline{\bar{e}}_{(\mathrm{i}) 56}=\overline{\bar{e}}_{(\mathrm{ii}) 82}=\overline{\bar{e}}_{(\mathrm{ii}) 82}^{+}=\overline{\bar{e}}_{(\mathrm{ii}) 65}^{+}=\overline{\bar{e}}_{(\mathrm{iii}) 724}=\overline{\bar{e}}_{(\mathrm{iv}) 1}=$ $\overline{\bar{e}}_{(\mathrm{iv}) 5}=\overline{\bar{e}}_{(\mathrm{iv}) 1}^{+}=\overline{\bar{e}}_{(\mathrm{iv}) 5}^{+}=\overline{\bar{e}}_{(\mathrm{v}) 35}^{-}=0$. The obtained results of the comprehensive inclusion-based indices are given by: $\overline{\overline{C I}}\left(\tilde{A}_{1}\right)=0.1783, \overline{\overline{C I}}\left(\tilde{A}_{2}\right)=0.3472, \overline{\overline{C I}}\left(\tilde{A}_{3}\right)=0.3326$, and $\overline{\overline{C I}}\left(\tilde{A}_{4}\right)=$ 0.2876 . The ranking order of the candidate bridge construction methods under inconsistent weight information is $A_{2} \succ A_{3} \succ A_{4} \succ A_{1}$. Therefore, the best choice is the incremental launching method $\left(A_{2}\right)$. It is telling that this result is significantly different from the ranking order yielded under consistent weight information. This is because the obtained results under consistent and inconsistent preference structures have distinct weight distributions in relation to the eight criteria. 


\subsection{Comparative analysis and discussions}

In the following, we conducted a comparative analysis to examine the results of the present inclusion-based method with those of the other approaches. First, because our proposed method originates from the main structure of TOPSIS, a well-known and widely used compromising method in MCDA, we chose it to be the foremost comparative method. Second, the proposed method was compared to Li's (2011c) closeness coefficient-based nonlinear programming method. Li (2011c) propounded a seminal study that applied IVIFSs to decision analysis using auxiliary nonlinear programming models and closeness IFSs. This research plays a significant role in the enrichment of decision modeling in interval-valued intuitionistic fuzzy settings. Therefore, we consider the obtained results by using Li's (2011c) method as a comparative benchmark. To compare the solution results on a common basis, we based the analysis on the same input data.

The first comparative method is the fuzzy TOPSIS approach. The basic concept of the TOPSIS method is that the chosen alternative should have the shortest distance from the positive ideal solution and the farthest distance from the negative ideal solution. For measuring distances between IVIFSs, we employed the generalization of Euclidean distances. The weighted Euclidean distances, $d\left(\tilde{A}_{i}, \tilde{A}_{*}\right)$ and $d\left(\tilde{A}_{i}, \tilde{A}_{-}\right)$, of each alternative from the interval-valued intuitionistic fuzzy positive-ideal and negative-ideal solutions, respectively, are derived from:

$$
\begin{aligned}
& d\left(\tilde{A}_{i}, \tilde{A}_{\star}\right)=\sqrt{\frac{1}{4} \sum_{j=1}^{n} w_{j}\left[\left(\mu_{i j}^{-}-\mu_{\star_{j}}^{-}\right)^{2}+\left(\mu_{i j}^{+}-\mu_{\star_{j}}^{+}\right)^{2}+\left(v_{i j}^{-}-v_{\star_{j}}^{-}\right)^{2}+\left(v_{i j}^{+}-v_{\star_{j}}^{+}\right)^{2}\right]}, \\
& d\left(\tilde{A}_{i}, \tilde{A}_{-}\right)=\sqrt{\frac{1}{4} \sum_{j=1}^{n} w_{j}\left[\left(\mu_{i j}^{-}-\mu_{-j}^{-}\right)^{2}+\left(\mu_{i j}^{+}-\mu_{-j}^{+}\right)^{2}+\left(v_{i j}^{-}-v_{-j}^{-}\right)^{2}+\left(v_{i j}^{+}-v_{-j}^{+}\right)^{2}\right]} .
\end{aligned}
$$

Consider $d\left(\tilde{A}_{2}, \tilde{A}_{*}\right)$ for example:

$$
\begin{aligned}
d\left(\tilde{A}_{2}, \tilde{A}_{\star}\right)= & \left(\frac { 1 } { 4 } \left[\left((0.68-0.72)^{2}+(0.71-0.77)^{2}+(0.06-0.06)^{2}+(0.26-0.20)^{2}\right) w_{1}+\right.\right. \\
& \left((0.04-0.03)^{2}+(0.12-0.07)^{2}+(0.61-0.66)^{2}+(0.86-0.86)^{2}\right) w_{2}+ \\
& \left((0.09-0.05)^{2}+(0.26-0.16)^{2}+(0.33-0.63)^{2}+(0.46-0.75)^{2}\right) w_{3}+ \\
& \left((0.12-0.07)^{2}+(0.23-0.23)^{2}+(0.64-0.64)^{2}+(0.67-0.67)^{2}\right) w_{4}+ \\
& \left((0.37-0.64)^{2}+(0.39-0.67)^{2}+(0.26-0.15)^{2}+(0.29-0.29)^{2}\right) w_{5}+ \\
& \left((0.18-0.07)^{2}+(0.19-0.14)^{2}+(0.74-0.74)^{2}+(0.78-0.78)^{2}\right) w_{6}+ \\
& \left((0.49-0.04)^{2}+(0.66-0.15)^{2}+(0.18-0.83)^{2}+(0.26-0.84)^{2}\right) w_{7}+ \\
& \left.\left.\left((0.18-0.04)^{2}+(0.41-0.09)^{2}+(0.17-0.88)^{2}+(0.28-0.90)^{2}\right) w_{8}\right]\right)^{0.5}= \\
& \left(\frac { 1 } { 4 } \left(0.0088 w_{1}+0.0051 w_{2}+0.1857 w_{3}+0.0025 w_{4}+0.1634 w_{5}+0.0146 w_{6}+\right.\right. \\
& \left.\left.1.2215 w_{7}+1.0105 w_{8}\right)\right)^{0.5} \cdot
\end{aligned}
$$


Based on the weighted Euclidean distances, the closeness coefficient $C C\left(\tilde{A}_{i}\right)$ of the characteristics $\tilde{A}_{i}$ for the alternative $\mathrm{A}_{\mathrm{i}}$ is defined with the following general formula:

$$
C C\left(\tilde{A}_{i}\right)=\frac{d\left(\tilde{A}_{i}, \tilde{A}_{-}\right)}{d\left(\tilde{A}_{i}, \tilde{A}_{\star}\right)+d\left(\tilde{A}_{i}, \tilde{A}_{-}\right)},
$$

where $0 \leq C C\left(\tilde{A}_{i}\right) \leq 1$.

Applying the weighted Euclidean distances, we constructed a nonlinear programming model under the incomplete weight information condition as follows:

$$
\begin{aligned}
& \text { [M5] } \max \left\{\sum_{i=1}^{m} C C\left(\tilde{A}_{i}\right)=\sum_{i=1}^{m} \frac{d\left(\tilde{A}_{i}, \tilde{A}_{-}\right)}{d\left(\tilde{A}_{i}, \tilde{A}_{*}\right)+d\left(\tilde{A}_{i}, \tilde{A}_{-}\right)}\right\} \\
& \text {s.t. } \quad\left(w_{1}, w_{2}, \cdots, w_{n}\right) \in \Gamma .
\end{aligned}
$$

The solution results in [M5] yield the optimal weight vector $\bar{w}=\left(\bar{w}_{1}, \bar{w}_{2}, \cdots, \bar{w}_{n}\right)$ and the corresponding closeness coefficients. Subsequently, the preference order of alternatives would be ranked according to the descending order of $\overline{C C}\left(\tilde{A}_{i}\right)$. Moreover, the alternative with the highest $\overline{C C}\left(\tilde{A}_{i}\right)$ value will be the best choice.

In the IVIFS context, we applied the fuzzy TOPSIS method to solve the same bridge construction method selection problem under incomplete preference information. According to (37), (38), and the evaluation $\tilde{A}_{i j}$ in the decision matrix $\tilde{D}$ in Table 2, we applied [M5] to establish the following nonlinear programming model:

$$
\begin{aligned}
& \max \left\{\left(\frac { 1 } { 4 } \left(0 w_{1}+0 w_{2}+0.3101 w_{3}+0.0865 w_{4}+0.4659 w_{5}+1.1603 w_{6}+0.3627 w_{7}+\right.\right.\right. \\
&\left.\left.1.0933 w_{8}\right)\right)^{0.5} /\left[\left(\frac { 1 } { 4 } \left(0.5105 w_{1}+1.1462 w_{2}+0.0009 w_{3}+0.2028 w_{4}+0.0016 w_{5}+\right.\right.\right. \\
&\left.\left.0.0116 w_{6}+0.4746 w_{7}+0 w_{8}\right)\right)^{0.5}+\left(\frac { 1 } { 4 } \left(0 w_{1}+0 w_{2}+0.3101 w_{3}+\right.\right. \\
&\left.\left.\left.0.0865 w_{4}+0.4659 w_{5}+1.1603 w_{6}+0.3627 w_{7}+1.0933 w_{8}\right)\right)^{0.5}\right]+ \\
&\left(\frac { 1 } { 4 } \left(0.4025 w_{1}+1.0423 w_{2}+0.0925 w_{3}+0.3106 w_{4}+0.1637 w_{5}+1.2237 w_{6}+0 w_{7}+\right.\right. \\
&\left.\left.0.0324 w_{8}\right)\right)^{0.5} /\left[\left(\frac { 1 } { 4 } \left(0.0088 w_{1}+0.0051 w_{2}+0.1857 w_{3}+0.0025 w_{4}+0.1634 w_{5}+\right.\right.\right. \\
&\left.\left.0.0146 w_{6}+1.2215 w_{7}+1.0105 w_{8}\right)\right)^{0.5}+\left(\frac { 1 } { 4 } \left(0.4025 w_{1}+1.0423 w_{2}+0.0925 w_{3}+\right.\right. \\
&\left.\left.\left.0.3106 w_{4}+0.1637 w_{5}+1.2237 w_{6}+0 w_{7}+0.0324 w_{8}\right)\right)^{0.5}\right]+ \\
&\left(\frac { 1 } { 4 } \left(0.4632 w_{1}+1.0182 w_{2}+0.1743 w_{3}+0.0125 w_{4}+0.4659 w_{5}+0.3302 w_{6}+0.7974 w_{7}+\right.\right.
\end{aligned}
$$




$$
\begin{aligned}
& \left.\left.0.1630 w_{8}\right)\right)^{0.5} /\left[\left(\frac { 1 } { 4 } \left(0.0121 w_{1}+0.0100 w_{2}+0.0877 w_{3}+0.2422 w_{4}+0.0016 w_{5}+\right.\right.\right. \\
& \left.\left.0.4681 w_{6}+0.0537 w_{7}+0.4945 w_{8}\right)\right)^{0.5}+\left(\frac { 1 } { 4 } \left(0.4632 w_{1}+1.0182 w_{2}+0.1743 w_{3}+\right.\right. \\
& \left.\left.\left.0.0125 w_{4}+0.4659 w_{5}+0.3302 w_{6}+0.7974 w_{7}+0.1630 w_{8}\right)\right)^{0.5}\right]+ \\
& \left(\frac { 1 } { 4 } \left(0.0395 w_{1}+0.3882 w_{2}+0.0397 w_{3}+0.0454 w_{4}+0.0025 w_{5}+0 w_{6}+1.2215 w_{7}+\right.\right. \\
& \left.\left.0.6694 w_{8}\right)\right)^{0.5} /\left[\left(\frac { 1 } { 4 } \left(0.3020 w_{1}+0.2254 w_{2}+0.2187 w_{3}+0.1975 w_{4}+0.4822 w_{5}+\right.\right.\right. \\
& \left.\left.1.3219 w_{6}+0 w_{7}+0.0563 w_{8}\right)\right)^{0.5}+\left(\frac { 1 } { 4 } \left(0.0395 w_{1}+0.3882 w_{2}+0.0397 w_{3}+\right.\right. \\
& \left.\left.\left.\left.0.0454 w_{4}+0.0025 w_{5}+0 w_{6}+1.2215 w_{7}+0.6694 w_{8}\right)\right)^{0.5}\right]\right\}
\end{aligned}
$$

subject to

$$
\begin{aligned}
& w_{5} \geq w_{6}, 0.12 \geq w_{8}-w_{2} \geq 0.07, w_{7}-w_{2} \geq w_{2}-w_{4}, 0.20 \geq w_{1} \geq 0.15,0.16 \geq w_{5} \\
& \geq 0.11, w_{3} \geq 0.6 \cdot w_{5}, w_{1}+w_{2}+w_{3}+w_{4}+w_{5}+w_{6}+w_{7}+w_{8}=1, w_{j} \geq 0 \text { for all } j .
\end{aligned}
$$

The optimal objective value is 2.2254 , and the optimal weight vector $\bar{w}=(0.15,0.1140908$, $0.066,0,0.11,0,0.3758184,0.1840908)$. In addition, the corresponding closeness coefficients $\overline{C C}\left(\tilde{A}_{1}\right)=0.5073411, \overline{C C}\left(\tilde{A}_{2}\right)=0.3573352, \overline{C C}\left(\tilde{A}_{3}\right)=0.6868759$, and $\overline{C C}\left(\tilde{A}_{4}\right)=0.6738473$. Thus, the optimal order of the four bridge construction methods is $A_{3} \succ A_{4} \succ A_{1} \succ A_{2}$.

The models in (39) and (44) yielded the same ranking results of the alternatives. However, when the fuzzy TOPSIS approach was employed within the IVIFS environment, the nonlinear programming model in (44) is markedly complex and is very difficult to solve. The model in (44) was solved using the Global Solver from LINGO 12.0 run on an x64-based PC with an Intel Core i5 CPU and 8 G RAM. LINGO completed the solution in 13 hours and 14 minutes; in addition, the number of iterations totaled 112,435,420. In contrast, we need to solve only the simple linear programming model in (39) (with less than 1 second running time) when we applied the proposed interval-valued intuitionistic fuzzy MCDA method based on inclusion comparison possibilities. In addition, the resulting ranking orders of the alternatives do not change. When we used the proposed method instead of the fuzzy TOPSIS method for the bridge construction selection problem or other decision-making problems, the complicated nonlinear programming model in [M5] can be replaced with the linear programming model in [M2]. The optimization problem can be easily solved using the Simplex method, and the degree of computational complexity can be significantly reduced.

The second comparative method is the closeness coefficient-based nonlinear programming method (Li 2011c). Li (2011c) proposed a new nonlinear programming method for solving MCDA problems based on the concept of closeness coefficients. His developed closeness coefficient is defined as the ratio between the square of the weighted Euclidean distance between an alternative and the negative ideal solution and the sum of the squares of the weighted Euclidean distances between the alternative and the positive and negative 
ideal solutions. Then, $\mathrm{Li}$ (2011c) constructed auxiliary nonlinear programming models to estimate the closeness IFSs, which are used to determine the optimal degrees of membership for the alternative rankings.

In the following, we applied Li's (2011c) method to solve the bridge construction selection problem. Note that in $\mathrm{Li}$ (2011c), all benefit criteria evaluation values for the positiveand negative-ideal solutions were expressed in the IVIFS vectors as $(([1,1],[0,0]))_{1 \times n}$ and $(([0,0],[1,1]))_{1 \times n}$, respectively. For the sake of a consistent comparative basis, we employed (15) and (19) to identify the positive- and negative-ideal solutions, respectively. According to Li's (2011c) method, we constructed auxiliary nonlinear programming models for the four bridge construction methods. Let $C_{i}$ denote the closeness IFS of alternative $A_{i} \in A$, where its degree of membership is bounded in the interval $\left[C_{i}^{l}, C_{i}^{u}\right]$. Take the alternative $\mathrm{A}_{2}$ as an example. Two nonlinear programming models were established as follows:

$$
\begin{aligned}
\min \{ & C_{2}^{l}=\left[\left(0.40 w_{1}\right)^{2}+\left(-0.39 w_{2}\right)^{2}+\left(-0.27 w_{3}\right)^{2}+\left(-0.23 w_{4}\right)^{2}+\left(0.22 w_{5}\right)^{2}+\left(-0.08 w_{6}\right)^{2}+\right. \\
& \left(0.00 w_{7}\right)^{2}+\left(-0.18 w_{8}\right)^{2}+\left(-0.20 w_{1}\right)^{2}+\left(0.69 w_{2}\right)^{2}+\left(0.00 w_{3}\right)^{2}+\left(0.26 w_{4}\right)^{2}+ \\
& \left.\left(-0.33 w_{5}\right)^{2}+\left(0.64 w_{6}\right)^{2}+\left(0.00 w_{7}\right)^{2}+\left(0.00 w_{8}\right)^{2}\right] /\left[\left(0.40 w_{1}\right)^{2}+\left(-0.39 w_{2}\right)^{2}+\right. \\
& \left(-0.27 w_{3}\right)^{2}+\left(-0.23 w_{4}\right)^{2}+\left(0.22 w_{5}\right)^{2}+\left(-0.08 w_{6}\right)^{2}+\left(0.00 w_{7}\right)^{2}+\left(-0.18 w_{8}\right)^{2}+ \\
& \left(-0.20 w_{1}\right)^{2}+\left(0.69 w_{2}\right)^{2}+\left(0.00 w_{3}\right)^{2}+\left(0.26 w_{4}\right)^{2}+\left(-0.33 w_{5}\right)^{2}+\left(0.64 w_{6}\right)^{2}+ \\
& \left(0.00 w_{7}\right)^{2}+\left(0.00 w_{8}\right)^{2}+\left(-0.04 w_{1}\right)^{2}+\left(0.01 w_{2}\right)^{2}+\left(0.04 w_{3}\right)^{2}+\left(0.05 w_{4}\right)^{2}+ \\
& \left(-0.27 w_{5}\right)^{2}+\left(0.11 w_{6}\right)^{2}+\left(0.45 w_{7}\right)^{2}+\left(0.14 w_{8}\right)^{2}+\left(0.06 w_{1}\right)^{2}+\left(0.00 w_{2}\right)^{2}+ \\
& \left.\left.\left(-0.29 w_{3}\right)^{2}+\left(0.00 w_{4}\right)^{2}+\left(0.00 w_{5}\right)^{2}+\left(0.00 w_{6}\right)^{2}+\left(-0.58 w_{7}\right)^{2}+\left(-0.62 w_{8}\right)^{2}\right]\right\}
\end{aligned}
$$

subject to

$w_{5} \geq w_{6}, 0.12 \geq w_{8}-w_{2} \geq 0.07, w_{7}-w_{2} \geq w_{2}-w_{4}, 0.20 \geq w_{1} \geq 0.15,0.16 \geq w_{5} \geq 0.11$, $w_{3} \geq 0.6 \cdot w_{5}, w_{1}+w_{2}+w_{3}+w_{4}+w_{5}+w_{6}+w_{7}+w_{8}=1, w_{j} \geq 0$ for all $j$,

$\max \left\{C_{2}^{u}=\left[\left(0.36 w_{1}\right)^{2}+\left(-0.46 w_{2}\right)^{2}+\left(-0.14 w_{3}\right)^{2}+\left(-0.26 w_{4}\right)^{2}+\left(0.08 w_{5}\right)^{2}+\left(-0.66 w_{6}\right)^{2}+\right.\right.$ $\left(0.00 w_{7}\right)^{2}+\left(0.00 w_{8}\right)^{2}+\left(-0.27 w_{1}\right)^{2}+\left(0.45 w_{2}\right)^{2}+\left(0.00 w_{3}\right)^{2}+\left(0.35 w_{4}\right)^{2}+$ $\left.\left(0.00 w_{5}\right)^{2}+\left(0.61 w_{6}\right)^{2}+\left(0.00 w_{7}\right)^{2}+\left(0.00 w_{8}\right)^{2}\right] /\left[\left(0.36 w_{1}\right)^{2}+\left(-0.46 w_{2}\right)^{2}+\right.$ $\left(-0.14 w_{3}\right)^{2}+\left(-0.26 w_{4}\right)^{2}+\left(0.08 w_{5}\right)^{2}+\left(-0.66 w_{6}\right)^{2}+\left(0.00 w_{7}\right)^{2}+\left(0.00 w_{8}\right)^{2}+$ $\left(-0.27 w_{1}\right)^{2}+\left(0.45 w_{2}\right)^{2}+\left(0.00 w_{3}\right)^{2}+\left(0.35 w_{4}\right)^{2}+\left(0.00 w_{5}\right)^{2}+\left(0.61 w_{6}\right)^{2}+$ $\left(0.00 w_{7}\right)^{2}+\left(0.00 w_{8}\right)^{2}+\left(-0.06 w_{1}\right)^{2}+\left(0.05 w_{2}\right)^{2}+\left(0.10 w_{3}\right)^{2}+\left(0.00 w_{4}\right)^{2}+$ $\left(-0.28 w_{5}\right)^{2}+\left(0.05 w_{6}\right)^{2}+\left(0.51 w_{7}\right)^{2}+\left(0.32 w_{8}\right)^{2}+\left(0.00 w_{1}\right)^{2}+\left(-0.05 w_{2}\right)^{2}+$ $\left.\left.\left(-0.30 w_{3}\right)^{2}+\left(0.00 w_{4}\right)^{2}+\left(0.11 w_{5}\right)^{2}+\left(0.00 w_{6}\right)^{2}+\left(-0.65 w_{7}\right)^{2}+\left(-0.71 w_{8}\right)^{2}\right]\right\}$ subject to

$w_{5} \geq w_{6}, 0.12 \geq w_{8}-w_{2} \geq 0.07, w_{7}-w_{2} \geq w_{2}-w_{4}, 0.20 \geq w_{1} \geq 0.15,0.16 \geq w_{5} \geq 0.11$, $w_{3} \geq 0.6 \cdot w_{5}, w_{1}+w_{2}+w_{3}+w_{4}+w_{5}+w_{6}+w_{7}+w_{8}=1, w_{j} \geq 0$ for all $j$. 
We obtained the optimal weight vectors $(0.15,0,0.066,0,0.11,0,0.604,0.07)$ and $(0.15$, $0,0.066,0.604,0.11,0,0,0.07)$ by solving (45) and (46), respectively. The optimal objective values of (45) and (46) are 0.03326215 and 0.9417259 , respectively. Then, the closeness IFS of alternative $A_{2}$ is $C_{2}=[0.03326215,0.9417259]$. In the same way, we acquired the other closeness IFSs as follows: $C_{1}=[0.1596862,0.8584206], C_{3}=[0.1849206,0.9429694]$, and $C_{4}=[0.04181132,0.9800826]$.

The inclusion comparison possibilities for the pair-wise alternatives in A were obtained and expressed in matrix format as follows:

$$
P=\left[\begin{array}{llll}
0.5 & 0.5134 & 0.4623 & 0.4988 \\
0.4866 & 0.5 & 0.4541 & 0.4873 \\
0.5377 & 0.5459 & 0.5 & 0.5312 \\
0.5012 & 0.5127 & 0.4688 & 0.5
\end{array}\right]
$$

The optimal membership degrees were computed as follows: $0.2479,0.2440,0.2596$, and 0.2486 for $A_{1}, A_{2}, A_{3}$, and $A_{4}$, respectively. Accordingly, the ranking order of the four bridge construction methods is $A_{3} \succ A_{4} \succ A_{1} \succ A_{2}$. Therefore, we can conclude that the ranking result yielded by the proposed method is the same as the benchmark result from Li's method. The validity of the final result yielded by the proposed method has been examined through the comparative analysis. Additionally, it is striking that after minimizing the tedious computational requirements, the proposed methodology can still generate credible solution results in this particular bridge construction selection problem. This comparative analysis demonstrated the potential of the proposed method to practical applications.

\section{Conclusions}

In the context of an IVIFS framework, this paper proposed an inclusion comparison-based method for solving multiple criteria decision-making problems under incomplete preference information. More specifically, this paper makes several important contributions to the existing literature on MCDA methodology based on IVIFSs. First, we extended the concept of inclusion comparison probability to propose lower and upper inclusion comparison possibilities defined on IVIFSs. Second, we defined an inclusion comparison possibility within the interval-valued intuitionistic fuzzy decision environment and discussed several important properties. Third, we propounded an inclusion-based index under considerations of displaced positive-ideal and negative-ideal solutions. Fourth, instead of relying on a complicated computational process to handle IVIFS data, we developed a simple and effective MCDA method based on the weighted inclusion-based indices. Finally, the proposed inclusion comparison approach is a flexible method capable of tackling decision-making problems that feature either consistent or inconsistent information regarding criterion importance under an incomplete preference structure. Furthermore, we illustrated an executive procedure of the proposed method by applying it to the selection problem of bridge construction methods. As depicted in the comparison analysis with the fuzzy TOPSIS approach, the proposed inclusion comparison-based method does not require complicated computational procedures, but 
still yields a credible solution. Therefore, we demonstrated the applicability of the proposed method to a real-world problem.

\section{Acknowledgements}

The author is very grateful to the respected editor and the anonymous referees for their insightful and constructive comments, which helped to improve the overall quality of the paper. This research is financially supported by the Taiwan Ministry of Science and Technology (Grant No. MOST 102-2410-H-182-013-MY3) and Chang Gung Memorial Hospital (BMRP 574).

\section{References}

Atanassov, K. T. 1983. Intuitionistic fuzzy sets, in Seventh Scientific Session of ITKR, June 1983, Sofia, Bulgaria Dep. in CINTI, Nd 1697/84.

Atanassov, K. T. 1986. Intuitionistic fuzzy sets, Fuzzy Sets and Systems 20(1): 87-96. http://dx.doi.org/10.1016/S0165-0114(86)80034-3

Atanassov, K. T. 2005. Answer to D. Dubois, S. Gottwald, P. Hajek, J. Kacprzyk and H. Prade's paper "Terminological difficulties in fuzzy set theory - the case of "Intuitionistic Fuzzy Sets"', Fuzzy Sets and Systems 156(3): 496-499. http://dx.doi.org/10.1016/j.fss.2005.06.003

Atanassov, K. T.; Gargov, G. 1989. Interval-valued intuitionistic fuzzy sets, Fuzzy Sets and Systems 31(3): 343-349. http://dx.doi.org/10.1016/0165-0114(89)90205-4

Chen, T.-Y.; Wang, H.-P.; Lu, Y.-Y. 2011. A multicriteria group decision-making approach based on interval-valued intuitionistic fuzzy sets: a comparative perspective, Expert Systems with Applications 38(6): 7647-7658. http://dx.doi.org/10.1016/j.eswa.2010.12.096

Chen, Z.; Yang, W. 2011. A new multiple attribute group decision making method in intuitionistic fuzzy setting, Applied Mathematical Modelling 35(9): 4424-4437. http://dx.doi.org/10.1016/j.apm.2011.03.015

Chen, Z.; Yang, W. 2012. A new multiple criteria decision making method based on intuitionistic fuzzy information, Expert Systems with Applications 39(4): 4328-4334. http://dx.doi.org/10.1016/j.eswa.2011.09.099

Deschrijver, G.; Kerre, E. E. 2007. On the position of intuitionistic fuzzy set theory in the framework of theories modeling imprecision, Information Sciences 177(8): 1860-1866. http://dx.doi.org/10.1016/j.ins.2006.11.005

Dubois, D.; Gottwald, S.; Hajek, P.; Kacprzyk, J.; Prade, H. 2005. Terminological difficulties in fuzzy set theory-the case of "intuitionistic fuzzy sets", Fuzzy Sets and Systems 156(3): 485-491. http://dx.doi.org/10.1016/j.fss.2005.06.001

Han, Z.; Liu, P. 2011. A fuzzy multi-attribute decision-making method under risk with unknown attribute weights, Technological and Economic Development of Economy 17(2): 246-258. http://dx.doi.org/10.3846/20294913.2011.580575

Li, D.-F. 2005. Multiattribute decision making models and methods using intuitionistic fuzzy sets, Journal of Computer and System Sciences 70(1): 73-85. http://dx.doi.org/10.1016/j.jcss.2004.06.002

Li, D.-F. 2010a. Multiattribute decision making method based on generalized OWA operators with intuitionistic fuzzy sets, Expert Systems with Applications 37(12): 8673-8678.

http://dx.doi.org/10.1016/j.eswa.2010.06.062

Li, D.-F. 2010b. TOPSIS-based nonlinear-programming methodology for multiattribute decision making with interval-valued intuitionistic fuzzy sets, IEEE Transactions on Fuzzy Systems 18(2): 299-311. http://dx.doi.org/10.1109/TFUZZ.2010.2041009 
Li, D.-F. 2010c. Mathematical-programming approach to matrix games with payoffs represented by Atanassov's interval-valued intuitionistic fuzzy sets, IEEE Transactions on Fuzzy Systems 18(6): 1112-1128. http://dx.doi.org/10.1109/TFUZZ.2010.2065812

Li, D.-F. 2010d. Linear programming method for MADM with interval-valued intuitionistic fuzzy sets, Expert Systems with Applications 37(8): 5939-5945. http://dx.doi.org/10.1016/j.eswa.2010.02.011

Li, D.-F. 2011a. The GOWA operator based approach to multiattribute decision making using intuitionistic fuzzy sets, Mathematical and Computer Modelling 53(5-6): 1182-1196. http://dx.doi.org/10.1016/j.mcm.2010.11.088

Li, D.-F. 2011b. Extension principles for interval-valued intuitionistic fuzzy sets and algebraic operations, Fuzzy Optimization and Decision Making 10(1): 45-58. http://dx.doi.org/10.1007/s10700-010-9095-9

Li, D.-F. 2011c. Closeness coefficient based nonlinear programming method for interval-valued intuitionistic fuzzy multiattribute decision making with incomplete preference information, Applied Soft Computing 11(4): 3402-3418. http://dx.doi.org/10.1016/j.asoc.2011.01.011

Li, D.-F. 2012a. Crucial and unsolved problems on Atanassov's intuitionistic fuzzy sets, Advances in Fuzzy Sets and Systems 11(1): 57-64.

Li, D.-F. 2012b. Representation theorem and extension principles for Atanassov's intuitionistic fuzzy sets and algebraic operations, Advances in Fuzzy Sets and Systems 11(2): 91-103.

Li, D.-F.; Chen, G.-H.; Huang, Z.-G. 2010. Linear programming method for multiattribute group decision making using IF sets, Information Sciences 180(9): 1591-1609.

http://dx.doi.org/10.1016/j.ins.2010.01.017

Nayagam, V. L. G.; Muralikrishnan, S.; Sivaraman, G. 2011. Multi-criteria decision-making method based on interval-valued intuitionistic fuzzy sets, Expert Systems with Applications 38(3): 1464-1467. http://dx.doi.org/10.1016/j.eswa.2010.07.055

Park, D. G.; Kwun, Y. C.; Park, J. H.; Park, I. Y. 2009. Correlation coefficient of interval-valued intuitionistic fuzzy sets and its application to multiple attribute group decision making problems, Mathematical and Computer Modelling 50(9-10): 1279-1293. http://dx.doi.org/10.1016/j.mcm.2009.06.010

Park, J. H.; Park, I. Y.; Kwun, Y. C.; Tan, X. 2011. Extension of the TOPSIS method for decision making problems under interval-valued intuitionistic fuzzy environment, Applied Mathematical Modelling 35(5): 2544-2556. http://dx.doi.org/10.1016/j.apm.2010.11.025

Park, K. S. 2004. Mathematical programming models for characterizing dominance and potential optimality when multicriteria alternative values and weights are simultaneously incomplete, IEEE Transactions on Systems, Man, and Cybernetics - Part A: Systems and Humans 34(5): 601-614. http://dx.doi.org/10.1109/TSMCA.2004.832828

Szmidt, E.; Kacprzyk, J. 2001. Entropy for intuitionistic fuzzy sets, Fuzzy Sets and Systems 118(3): 467-477. http://dx.doi.org/10.1016/S0165-0114(98)00402-3

Tan, C. 2011. A multi-criteria interval-valued intuitionistic fuzzy group decision making with Choquet integral-based TOPSIS, Expert Systems with Applications 38(4): 3023-3033. http://dx.doi.org/10.1016/j.eswa.2010.08.092

Wang, L.-L.; Li, D.-F.; Zhang, S.-S. 2013. Mathematical programming methodology for multiattribute decision making using interval-valued intuitionistic fuzzy sets, Journal of Intelligent and Fuzzy Systems 24(4): 755-763.

Wei, G.; Zhao, X. 2012. Some induced correlated aggregating operators with intuitionistic fuzzy information and their application to multiple attribute group decision making, Expert Systems with Applications 39(2): 2026-2034. http://dx.doi.org/10.1016/j.eswa.2011.08.031

Wei, G.; Zhao, X.; Wang, H. 2012. An approach to multiple attribute group decision making with interval intuitionistic trapezoidal fuzzy information, Technological and Economic Development of Economy 18(2): 317-330. http://dx.doi.org/10.3846/20294913.2012.676995 
Wei, G.-W.; Wang, H.-J.; Lin, R. 2011. Application of correlation coefficient to interval-valued intuitionistic fuzzy multiple attribute decision-making with incomplete weight information, Knowledge and Information Systems 26(2): 337-349. http://dx.doi.org/10.1007/s10115-009-0276-1

$\mathrm{Xu}, \mathrm{Z}$. S.; Chen, J. 2008. MAGDM linear-programming models with distinct uncertain preference structures, IEEE Transactions on Systems, Man, and Cybernetics - Part B: Cybernetics 38(5): 1356-1370. http://dx.doi.org/10.1109/TSMCB.2008.925752

Ye, J. 2012. Multicriteria decision-making method using the Dice similarity measure based on the reduct intuitionistic fuzzy sets of interval-valued intuitionistic fuzzy sets, Applied Mathematical Modelling 36(9): 4466-4472. http://dx.doi.org/10.1016/j.apm.2011.11.075

Zhang, X.; Liu, P. D. 2010. Method for aggregating triangular fuzzy intuitionistic fuzzy information and its application to decision making, Technological and Economic Development of Economy 16(2): 280-290. http://dx.doi.org/10.3846/tede.2010.18

\section{APPENDIX I}

In this appendix, we will verify the four properties (P1.1)-(P1.4) of the lower and upper inclusion comparison possibilities previously mentioned in Property 1.

\section{Proof of (P1.1)}

It is obvious that:

$$
\max \left\{\frac{\left(1-v_{\rho^{\prime} j}^{-}\right)-\mu_{\rho j}^{-}}{\left(1-\mu_{\rho j}^{-}-v_{\rho j}^{+}\right)+\left(1-\mu_{\rho^{\prime} j}^{+}-v_{\rho^{\prime} j}^{-}\right)}, 0\right\} \geq 0 .
$$

It follows that:

$$
1-\max \left\{\frac{\left(1-v_{\rho^{\prime} j}^{-}\right)-\mu_{\rho j}^{-}}{\left(1-\mu_{\rho j}^{-}-v_{\rho j}^{+}\right)+\left(1-\mu_{\rho^{\prime} j}^{+}-v_{\rho^{\prime} j}^{-}\right)}, 0\right\} \leq 1 .
$$

Furthermore,

$$
0 \leq \max \left\{1-\max \left\{\frac{\left(1-v_{\rho^{\prime} j}^{-}\right)-\mu_{\rho j}^{-}}{\left(1-\mu_{\rho j}^{-}-v_{\rho j}^{+}\right)+\left(1-\mu_{\rho^{\prime} j}^{+}-v_{\rho^{\prime} j}^{-}\right)}, 0\right\}, 0\right\} \leq 1 .
$$

Therefore, we know that $0 \leq p^{-}\left(\tilde{A}_{\rho j} \supseteq \tilde{A}_{\rho^{\prime} j}\right) \leq 1$ is satisfied, i.e., (P1.1) is valid.

\section{Proof of (P1.2)}

(P1.2) is proven by analogy to (P1.1).

\section{Proof of (P1.3)}

Because $\mu_{\rho j}^{-} \leq \mu_{\rho j}^{+}$and $v_{\rho^{\prime} j}^{-} \leq v_{\rho^{\prime} j}^{+}$, we obtain:

$$
\left(1-v_{\rho^{\prime} j}^{-}\right)-\mu_{\rho j}^{-} \geq\left(1-v_{\rho^{\prime} j}^{+}\right)-\mu_{\rho j}^{+} .
$$


Considering the denominator in (11), we have:

$$
\begin{aligned}
& \left(1-\mu_{\rho j}^{-}-v_{\rho j}^{+}\right)+\left(1-\mu_{\rho^{\prime} j}^{+}-v_{\rho^{\prime} j}^{-}\right)= \\
& \left(\mu_{\rho j}^{+}-\mu_{\rho j}^{-}+1-\mu_{\rho j}^{+}-v_{\rho j}^{+}\right)+\left(v_{\rho^{\prime} j}^{+}-v_{\rho^{\prime} j}^{-}+1-\mu_{\rho^{\prime} j}^{+}-v_{\rho^{\prime} j}^{+}\right)= \\
& \left(\mu_{\rho j}^{+}-\mu_{\rho j}^{-}\right)+\left(v_{\rho^{\prime} j}^{+}-v_{\rho^{\prime} j}^{-}\right)+\left[\left(1-\mu_{\rho j}^{+}-v_{\rho j}^{+}\right)+\left(1-\mu_{\rho^{\prime} j}^{+}-v_{\rho^{\prime} j}^{+}\right)\right] .
\end{aligned}
$$

With respect to the denominator in (12), we obtain:

$$
\begin{aligned}
& \left(1-\mu_{\rho j}^{+}-v_{\rho j}^{-}\right)+\left(1-\mu_{\rho^{\prime} j}^{-}-v_{\rho^{\prime} j}^{+}\right)= \\
& \left(v_{\rho j}^{+}-v_{\rho j}^{-}\right)+\left(\mu_{\rho^{\prime} j}^{+}-\mu_{\rho^{\prime} j}^{-}\right)+\left[\left(1-\mu_{\rho j}^{+}-v_{\rho j}^{+}\right)+\left(1-\mu_{\rho^{\prime} j}^{+}-v_{\rho^{\prime} j}^{+}\right)\right] .
\end{aligned}
$$

We can observe that $\left[\left(1-\mu_{\rho j}^{+}-v_{\rho j}^{+}\right)+\left(1-\mu_{\rho^{\prime} j}^{+}-v_{\rho^{\prime} j}^{+}\right)\right]$is a common term in (A2) and (A3). Therefore, the comparison of $\left(1-\mu_{\rho j}^{-}-v_{\rho j}^{+}\right)+\left(1-\mu_{\rho^{\prime} j}^{+}-v_{\rho^{\prime} j}^{-}\right)$and $\left(1-\mu_{\rho j}^{+}-\right.$ $\left.v_{\rho j}^{-}\right)+\left(1-\mu_{\rho^{\prime} j}^{-}-v_{\rho^{\prime} j}^{+}\right)$can be directly conducted via $\left(\mu_{\rho j}^{+}-\mu_{\rho j}^{-}\right)+\left(v_{\rho^{\prime} j}^{+}-v_{\rho^{\prime} j}^{-}\right)$and $\left(v_{\rho j}^{+}-v_{\rho j}^{-}\right)+\left(\mu_{\rho^{\prime} j}^{+}-\mu_{\rho^{\prime} j}^{-}\right)$. In the following, we will verify (P1.3) with regard to the conditions of (a) $\left(\mu_{\rho j}^{+}-\mu_{\rho j}^{-}\right)+\left(v_{\rho^{\prime} j}^{+}-v_{\rho^{\prime} j}^{-}\right) \leq\left(v_{\rho j}^{+}-v_{\rho j}^{-}\right)+\left(\mu_{\rho^{\prime} j}^{+}-\mu_{\rho^{\prime} j}^{-}\right)$and (b) $\left(\mu_{\rho j}^{+}-\mu_{\rho j}^{-}\right)+\left(v_{\rho^{\prime} j}^{+}-v_{\rho^{\prime} j}^{-}\right) \geq\left(v_{\rho j}^{+}-v_{\rho j}^{-}\right)+\left(\mu_{\rho^{\prime} j}^{+}-\mu_{\rho^{\prime} j}^{-}\right)$.

According to (A1) and Condition (a), it follows that:

$$
\frac{\left(1-v_{\rho^{\prime} j}^{-}\right)-\mu_{\rho j}^{-}}{\left(1-\mu_{\rho j}^{-}-v_{\rho j}^{+}\right)+\left(1-\mu_{\rho^{\prime} j}^{+}-v_{\rho^{\prime} j}^{-}\right)} \geq \frac{\left(1-v_{\rho^{\prime} j}^{+}\right)-\mu_{\rho j}^{+}}{\left(1-\mu_{\rho j}^{+}-v_{\rho j}^{-}\right)+\left(1-\mu_{\rho^{\prime} j}^{-}-v_{\rho^{\prime} j}^{+}\right)} .
$$

Therefore, it is proven that $p^{-}\left(\tilde{A}_{\rho j} \supseteq \tilde{A}_{\rho^{\prime} j}\right) \leq p^{+}\left(\tilde{A}_{\rho j} \supseteq \tilde{A}_{\rho^{\prime} j}\right)$ in Condition (a).

Regarding the proof in Condition (b), we know that $0 \leq \mu_{\rho j}^{+}-\mu_{\rho j}^{-} \leq \mu_{\rho j}^{+}, 0 \leq v_{\rho^{\prime} j}^{+}-v_{\rho^{\prime} j}^{-} \leq v_{\rho^{\prime} j}^{+}$, $0 \leq v_{\rho j}^{+}-v_{\rho j}^{-} \leq v_{\rho j}^{+}$, and $0 \leq \mu_{\rho^{\prime} j}^{+}-\mu_{\rho^{\prime} j}^{-} \leq \mu_{\rho^{\prime} j}^{+}$. Consider the special cases of $\mu_{\rho j}^{-}=0, v_{\rho^{\prime} j}^{-}=0$, $v_{\rho j}^{-}=v_{\rho j}^{+}$, and $\mu_{\rho^{\prime} j}^{-}=\mu_{\rho^{\prime} j}^{+}$, in which (A2) has the maximal value and (A3) has the minimal value. Here, the fraction in (11) becomes:

$$
\frac{(1-0)-0}{\left(1-0-v_{\rho j}^{+}\right)+\left(1-\mu_{\rho^{\prime} j}^{+}-0\right)}=\frac{1}{\left(1-v_{\rho j}^{+}\right)+\left(1-\mu_{\rho^{\prime} j}^{+}\right)} \geq \frac{1}{2},
$$

because $1-v_{\rho j}^{+} \leq 1$ and $1-\mu_{\rho^{\prime} j}^{+} \leq 1$.

Next, considering the conditions where $\mu_{\rho j}^{+}-\mu_{\rho j}^{-} \in\left[0, \mu_{\rho j}^{+}\right], v_{\rho^{\prime} j}^{+}-v_{\rho^{\prime} j}^{-} \in\left[0, v_{\rho^{\prime} j}^{+}\right]$, $v_{\rho j}^{+}-v_{\rho j}^{-} \in\left[0, v_{\rho j}^{+}\right]$, and $\mu_{\rho^{\prime} j}^{+}-\mu_{\rho^{\prime} j}^{-} \in\left[0, \mu_{\rho^{\prime} j}^{+}\right]$, we obtain that $\mu_{\rho j}^{+}+v_{\rho^{\prime} j}^{+} \geq v_{\rho j}^{+}+\mu_{\rho^{\prime} j}^{+}$from $\left(\mu_{\rho j}^{+}-\mu_{\rho j}^{-}\right)+\left(v_{\rho^{\prime} j}^{+}-v_{\rho^{\prime} j}^{-}\right) \geq\left(v_{\rho j}^{+}-v_{\rho j}^{-}\right)+\left(\mu_{\rho^{\prime} j}^{+}-\mu_{\rho^{\prime} j}^{-}\right)$in Condition (b). Therefore, this implies that $1-\mu_{\rho j}^{+}-v_{\rho^{\prime} j}^{+} \leq 1-v_{\rho j}^{+}-\mu_{\rho^{\prime} j}^{+}$. Following the discussion above, the fraction in (12) becomes: 


$$
\begin{aligned}
& \frac{\left(1-v_{\rho^{\prime} j}^{+}\right)-\mu_{\rho j}^{+}}{\left(1-\mu_{\rho j}^{+}-v_{\rho j}^{+}\right)+\left(1-\mu_{\rho^{\prime} j}^{+}-v_{\rho^{\prime} j}^{+}\right)}= \\
& \frac{1-\mu_{\rho j}^{+}-v_{\rho^{\prime} j}^{+}}{\left(1-\mu_{\rho j}^{+}-v_{\rho^{\prime} j}^{+}\right)+\left(1-v_{\rho j}^{+}-\mu_{\rho^{\prime} j}^{+}\right)} \leq \frac{1-\mu_{\rho j}^{+}-v_{\rho^{\prime} j}^{+}}{\left(1-\mu_{\rho j}^{+}-v_{\rho^{\prime} j}^{+}\right)+\left(1-\mu_{\rho j}^{+}-v_{\rho^{\prime} j}^{+}\right)}=\frac{1}{2} .
\end{aligned}
$$

Thereby, (A4) is not less than (A5). According to the comparison result in the case of the maximal value in (A2) and the minimal value in (A3), it directly follows that:

$$
\frac{\left(1-v_{\rho^{\prime} j}^{-}\right)-\mu_{\rho j}^{-}}{\left(1-\mu_{\rho j}^{-}-v_{\rho j}^{+}\right)+\left(1-\mu_{\rho^{\prime} j}^{+}-v_{\rho^{\prime} j}^{-}\right)} \geq \frac{\left(1-v_{\rho^{\prime} j}^{+}\right)-\mu_{\rho j}^{+}}{\left(1-\mu_{\rho j}^{+}-v_{\rho j}^{-}\right)+\left(1-\mu_{\rho^{\prime} j}^{-}-v_{\rho^{\prime} j}^{+}\right)} .
$$

It is easily proven that $p^{-}\left(\tilde{A}_{\rho j} \supseteq \tilde{A}_{\rho^{\prime} j}\right) \leq p^{+}\left(\tilde{A}_{\rho j} \supseteq \tilde{A}_{\rho^{\prime} j}\right)$ in Condition (b). Therefore, we prove that $(\mathrm{P} 1.3)$ is valid.

\section{Proof of (P1.4)}

Three cases are taken into consideration in this proof and include the following: (a) $1-v_{\rho^{\prime} j}^{-} \geq \mu_{\rho j}^{-}$ and $1-v_{\rho j}^{+} \geq \mu_{\rho^{\prime} j}^{+}$;(b) $1-v_{\rho^{\prime} j}^{-} \geq \mu_{\rho j}^{-}$and $1-v_{\rho j}^{+} \leq \mu_{\rho^{\prime} j}^{+}$; and (c) $1-v_{\rho^{\prime} j}^{-} \leq \mu_{\rho j}^{-}$and $1-v_{\rho j}^{+} \geq \mu_{\rho^{\prime} j}^{+}$. With regard to the conditions that $1-v_{\rho^{\prime} j}^{-} \leq \mu_{\rho j}^{-}$and $1-v_{\rho j}^{+} \leq \mu_{\rho^{\prime} j}^{+}$, we combine the two inequalities $\mu_{\rho j}^{-}+v_{\rho^{\prime} j}^{-} \geq 1$ and $v_{\rho j}^{+}+\mu_{\rho^{\prime} j}^{+} \geq 1$ to obtain $\mu_{\rho j}^{-}+v_{\rho j}^{+}+\mu_{\rho^{\prime} j}^{+}+v_{\rho^{\prime} j}^{-} \geq 2$. However, this result is not permitted because of the axioms $\mu_{\rho j}^{+}+v_{\rho j}^{+} \leq 1$ and $\mu_{\rho^{\prime} j}^{+}+v_{\rho^{\prime} j}^{+} \leq 1$, as indicated in Definition 3. Therefore, discussing the conditions of $1-v_{\rho^{\prime} j}^{-} \leq \mu_{\rho j}^{-}$and $1-v_{\rho j}^{+} \leq \mu_{\rho^{\prime} j}^{+}$is actually unnecessary.

In Case (a), because $1-\mu_{\rho j}^{-}-v_{\rho j}^{+} \geq 0,1-\mu_{\rho^{\prime} j}^{+}-v_{\rho^{\prime} j}^{-} \geq 0$, and $\left(1-v_{\rho^{\prime} j}^{-}\right)-\mu_{\rho j}^{-} \geq 0$, we know that:

$$
\begin{gathered}
\frac{\left(1-v_{\rho^{\prime} j}^{-}\right)-\mu_{\rho j}^{-}}{\left(1-\mu_{\rho j}^{-}-v_{\rho j}^{+}\right)+\left(1-\mu_{\rho^{\prime} j}^{+}-v_{\rho^{\prime} j}^{-}\right)} \geq 0, \text { and } \\
\max \left\{\frac{\left(1-v_{\rho^{\prime} j}^{-}\right)-\mu_{\rho j}^{-}}{\left(1-\mu_{\rho j}^{-}-v_{\rho j}^{+}\right)+\left(1-\mu_{\rho^{\prime} j}^{+}-v_{\rho^{\prime} j}^{-}\right)}, 0\right\}=\frac{\left(1-v_{\rho^{\prime} j}^{-}\right)-\mu_{\rho j}^{-}}{\left(1-\mu_{\rho j}^{-}-v_{\rho j}^{+}\right)+\left(1-\mu_{\rho^{\prime} j}^{+}-v_{\rho^{\prime} j}^{-}\right)} .
\end{gathered}
$$

Additionally, because $1-v_{\rho j}^{+} \geq \mu_{\rho^{\prime} j}^{+}$,

$$
1-\frac{\left(1-v_{\rho^{\prime} j}^{-}\right)-\mu_{\rho j}^{-}}{\left(1-\mu_{\rho j}^{-}-v_{\rho j}^{+}\right)+\left(1-\mu_{\rho^{\prime} j}^{+}-v_{\rho^{\prime} j}^{-}\right)}=\frac{1-v_{\rho j}^{+}-\mu_{\rho^{\prime} j}^{+}}{\left(1-\mu_{\rho j}^{-}-v_{\rho j}^{+}\right)+\left(1-\mu_{\rho^{\prime} j}^{+}-v_{\rho^{\prime} j}^{-}\right)} \geq 0 .
$$


Therefore, in Case (a), we obtain:

$$
p^{-}\left(\tilde{A}_{\rho j} \supseteq \tilde{A}_{\rho^{\prime} j}\right)=\frac{1-v_{\rho j}^{+}-\mu_{\rho^{\prime} j}^{+}}{\left(1-\mu_{\rho j}^{-}-v_{\rho j}^{+}\right)+\left(1-\mu_{\rho^{\prime} j}^{+}-v_{\rho^{\prime} j}^{-}\right)} .
$$

In a similar way, we obtain:

$$
p^{+}\left(\tilde{A}_{\rho^{\prime} j} \supseteq \tilde{A}_{\rho j}\right)=\frac{1-v_{\rho^{\prime} j}^{-}-\mu_{\rho j}^{-}}{\left(1-\mu_{\rho^{\prime} j}^{+}-v_{\rho^{\prime} j}^{-}\right)+\left(1-\mu_{\rho j}^{-}-v_{\rho j}^{+}\right)} .
$$

It is clear that $p^{-}\left(\tilde{A}_{\rho j} \supseteq \tilde{A}_{\rho^{\prime} j}\right)+p^{+}\left(\tilde{A}_{\rho^{\prime} j} \supseteq \tilde{A}_{\rho j}\right)=1$ follows from Case (a).

Next, consider the conditions in Case (b). Likewise, according to Case (b), we have:

$$
1-\max \left\{\frac{\left(1-v_{\rho^{\prime} j}^{-}\right)-\mu_{\rho j}^{-}}{\left(1-\mu_{\rho j}^{-}-v_{\rho j}^{+}\right)+\left(1-\mu_{\rho^{\prime} j}^{+}-v_{\rho^{\prime} j}^{-}\right)}, 0\right\}=\frac{1-v_{\rho j}^{+}-\mu_{\rho^{\prime} j}^{+}}{\left(1-\mu_{\rho j}^{-}-v_{\rho j}^{+}\right)+\left(1-\mu_{\rho^{\prime} j}^{+}-v_{\rho^{\prime} j}^{-}\right)} .
$$

Because $1-v_{\rho j}^{+} \leq \mu_{\rho^{\prime} j}^{+}$in Case (b), we obtain $p^{-}\left(\tilde{A}_{\rho j} \supseteq \tilde{A}_{\rho^{\prime} j}\right)=0$. Additionally, because $1-v_{\rho j}^{+} \leq \mu_{\rho^{\prime} j}^{+}$, we obtain:

$$
\begin{gathered}
\frac{\left(1-v_{\rho j}^{+}\right)-\mu_{\rho^{\prime} j}^{+}}{\left(1-\mu_{\rho^{\prime} j}^{+}-v_{\rho^{\prime} j}^{-}\right)+\left(1-\mu_{\rho j}^{-}-v_{\rho j}^{+}\right)} \leq 0, \text { and } \\
\max \left\{\frac{\left(1-v_{\rho j}^{+}\right)-\mu_{\rho^{\prime} j}^{+}}{\left(1-\mu_{\rho^{\prime} j}^{+}-v_{\rho^{\prime} j}^{-}\right)+\left(1-\mu_{\rho j}^{-}-v_{\rho j}^{+}\right)}, 0\right\}=0 .
\end{gathered}
$$

Thereby, $p^{+}\left(\tilde{A}_{\rho^{\prime} j} \supseteq \tilde{A}_{\rho j}\right)=1$. It is easily observed that $p^{-}\left(\tilde{A}_{\rho j} \supseteq \tilde{A}_{\rho^{\prime} j}\right)+p^{+}\left(\tilde{A}_{\rho^{\prime} j} \supseteq \tilde{A}_{\rho j}\right)=1$ in Case (b).

With regard to the condition that $1-v_{\rho^{\prime} j}^{-} \leq \mu_{\rho j}^{-}$in Case (c), we obtain:

$$
\frac{\left(1-v_{\rho^{\prime} j}^{-}\right)-\mu_{\rho j}^{-}}{\left(1-\mu_{\rho j}^{-}-v_{\rho j}^{+}\right)+\left(1-\mu_{\rho^{\prime} j}^{+}-v_{\rho^{\prime} j}^{-}\right)} \leq 0 .
$$

Therefore, $p^{-}\left(\tilde{A}_{\rho j} \supseteq \tilde{A}_{\rho^{\prime} j}\right)=1$. Moreover, with the condition that $1-v_{\rho j}^{+} \geq \mu_{\rho^{\prime} j}^{+}$,

$$
\frac{\left(1-v_{\rho j}^{+}\right)-\mu_{\rho^{\prime} j}^{+}}{\left(1-\mu_{\rho^{\prime} j}^{+}-v_{\rho^{\prime} j}^{-}\right)+\left(1-\mu_{\rho j}^{-}-v_{\rho j}^{+}\right)} \geq 0 .
$$


Applying the condition that $1-v_{\rho^{\prime} j}^{-} \leq \mu_{\rho j}^{-}$, we obtain:

$$
\begin{aligned}
p^{+}\left(\tilde{A}_{\rho^{\prime} j} \supseteq \tilde{A}_{\rho j}\right)= & \max \left\{1-\frac{\left(1-v_{\rho j}^{+}\right)-\mu_{\rho^{\prime} j}^{+}}{\left(1-\mu_{\rho^{\prime} j}^{+}-v_{\rho^{\prime} j}^{-}\right)+\left(1-\mu_{\rho j}^{-}-v_{\rho j}^{+}\right)}, 0\right\}= \\
& \max \left\{\frac{1-v_{\rho^{\prime} j}^{-}-\mu_{\rho j}^{-}}{\left(1-\mu_{\rho^{\prime} j}^{+}-v_{\rho^{\prime} j}^{-}\right)+\left(1-\mu_{\rho j}^{-}-v_{\rho j}^{+}\right)}, 0\right\}=0 .
\end{aligned}
$$

Accordingly, $p^{-}\left(\tilde{A}_{\rho j} \supseteq \tilde{A}_{\rho^{\prime} j}\right)+p^{+}\left(\tilde{A}_{\rho^{\prime} j} \supseteq \tilde{A}_{\rho j}\right)=1$ in Case (c). Therefore, we prove that (P1.4) is valid.

\section{APPENDIX II}

In this appendix, we will verify the four properties (P2.1)-(P2.6) of the inclusion comparison possibility previously mentioned in Property 2.

\section{Proof of (P2.1)}

According to (P1.1) and (P1.2) in Property 1, we know that $0 \leq p^{-}\left(\tilde{A}_{\rho j} \supseteq \tilde{A}_{\rho^{\prime} j}\right) \leq 1$ and $0 \leq p^{+}\left(\tilde{A}_{\rho j} \supseteq \tilde{A}_{\rho^{\prime} j}\right) \leq 1$. It is obvious that the arithmetic mean of $p^{-}\left(\tilde{A}_{\rho j} \supseteq \tilde{A}_{\rho^{\prime} j}\right)$ and $p^{+}\left(\tilde{A}_{\rho j} \supseteq \tilde{A}_{\rho^{\prime} j}\right)$ is between 0 and 1, i.e., $0 \leq p\left(\tilde{A}_{\rho j} \supseteq \tilde{A}_{\rho^{\prime} j}\right) \leq 1$. Thereby, (P2.1) is valid.

\section{Proof of (P2.2)}

Consider the lower inclusion comparison possibility $p^{-}\left(\tilde{A}_{\rho j} \supseteq \tilde{A}_{\rho^{\prime} j}\right)$ in the case of $1-v_{\rho j}^{-} \leq \mu_{\rho^{\prime} j}^{-}$. Because $v_{\rho j}^{-} \leq v_{\rho j}^{+}, \mu_{\rho^{\prime} j}^{-} \leq \mu_{\rho^{\prime} j}^{+}$, and $1-v_{\rho j}^{-}-\mu_{\rho^{\prime} j}^{-} \leq 0$, it easily follows that:

$$
1-v_{\rho j}^{+}-\mu_{\rho^{\prime} j}^{+} \leq 1-v_{\rho j}^{-}-\mu_{\rho^{\prime} j}^{-} \leq 0 .
$$

It directly implies that:

$$
\left(1-\mu_{\rho j}^{-}-v_{\rho j}^{+}\right)+\left(1-\mu_{\rho^{\prime} j}^{+}-v_{\rho^{\prime} j}^{-}\right)=\left(1-v_{\rho^{\prime} j}^{-}-\mu_{\rho j}^{-}\right)+\left(1-v_{\rho j}^{+}-\mu_{\rho^{\prime} j}^{+}\right) \leq 1-v_{\rho^{\prime} j}^{-}-\mu_{\rho j}^{-} \text {. }
$$

Then, we have:

$$
\frac{1-v_{\rho^{\prime} j}^{-}-\mu_{\rho j}^{-}}{\left(1-\mu_{\rho j}^{-}-v_{\rho j}^{+}\right)+\left(1-\mu_{\rho^{\prime} j}^{+}-v_{\rho^{\prime} j}^{-}\right)} \geq 1
$$

Therefore, $p^{-}\left(\tilde{A}_{\rho j} \supseteq \tilde{A}_{\rho^{\prime} j}\right)=0$.

Next, consider the upper inclusion comparison possibility $p^{+}\left(\tilde{A}_{\rho j} \supseteq \tilde{A}_{\rho^{\prime} j}\right)$ if $1-v_{\rho j}^{-} \leq \mu_{\rho^{\prime} j}^{-}$. Because $1-v_{\rho j}^{-}-\mu_{\rho^{\prime} j}^{-} \leq 0$, we obtain:

$$
\left(1-\mu_{\rho j}^{+}-v_{\rho j}^{-}\right)+\left(1-\mu_{\rho^{\prime} j}^{-}-v_{\rho^{\prime} j}^{+}\right)=\left(1-v_{\rho^{\prime} j}^{+}-\mu_{\rho j}^{+}\right)+\left(1-v_{\rho j}^{-}-\mu_{\rho^{\prime} j}^{-}\right) \leq 1-v_{\rho^{\prime} j}^{+}-\mu_{\rho j}^{+} \text {. }
$$


Furthermore,

$$
\frac{1-v_{\rho^{\prime} j}^{+}-\mu_{\rho j}^{+}}{\left(1-\mu_{\rho j}^{+}-v_{\rho j}^{-}\right)+\left(1-\mu_{\rho^{\prime} j}^{-}-v_{\rho^{\prime} j}^{+}\right)} \geq 1
$$

Thereby, $p^{+}\left(\tilde{A}_{\rho j} \supseteq \tilde{A}_{\rho^{\prime} j}\right)=0$. Therefore, $p\left(\tilde{A}_{\rho j} \supseteq \tilde{A}_{\rho^{\prime} j}\right)=0$ in the case of $1-v_{\rho j}^{-} \leq \mu_{\rho^{\prime} j}^{-}$. We prove that $(\mathrm{P} 2.2)$ is valid.

\section{Proof of (P2.3)}

Because $1-v_{\rho^{\prime} j}^{-}-\mu_{\rho j}^{-} \leq 0$, we have:

$$
\frac{1-v_{\rho^{\prime} j}^{-}-\mu_{\rho j}^{-}}{\left(1-\mu_{\rho j}^{-}-v_{\rho j}^{+}\right)+\left(1-\mu_{\rho^{\prime} j}^{+}-v_{\rho^{\prime} j}^{-}\right)} \leq 0 .
$$

Thereby, $p^{-}\left(\tilde{A}_{\rho j} \supseteq \tilde{A}_{\rho^{\prime} j}\right)=1$. Additionally, because $v_{\rho^{\prime} j}^{-} \leq v_{\rho^{\prime} j}^{+}, \mu_{\rho j}^{-} \leq \mu_{\rho j}^{+}$, and $1-v_{\rho^{\prime} j}^{-}$ $\mu_{\rho j}^{-} \leq 0$, we obtain $1-v_{\rho^{\prime} j}^{+}-\mu_{\rho j}^{+} \leq 1-v_{\rho^{\prime} j}^{-}-\mu_{\rho j}^{-} \leq 0$ and

$$
\frac{1-v_{\rho^{\prime} j}^{+}-\mu_{\rho j}^{+}}{\left(1-\mu_{\rho j}^{+}-v_{\rho j}^{-}\right)+\left(1-\mu_{\rho^{\prime} j}^{-}-v_{\rho^{\prime} j}^{+}\right)} \leq 0 .
$$

Therefore, $p^{+}\left(\tilde{A}_{\rho j} \supseteq \tilde{A}_{\rho^{\prime} j}\right)=1$. It follows that $p\left(\tilde{A}_{\rho j} \supseteq \tilde{A}_{\rho^{\prime} j}\right)=1$ if $\mu_{\rho j}^{-} \geq 1-v_{\rho^{\prime} j}^{-}$, i.e., (P2.3) is valid.

\section{Proof of (P2.4)}

According to Definition 4, we have:

$$
p\left(\tilde{A}_{\rho j} \supseteq \tilde{A}_{\rho^{\prime} j}\right)=\frac{1}{2}\left(p^{-}\left(\tilde{A}_{\rho j} \supseteq \tilde{A}_{\rho^{\prime} j}\right)+p^{+}\left(\tilde{A}_{\rho j} \supseteq \tilde{A}_{\rho^{\prime} j}\right)\right)
$$

and

$$
p\left(\tilde{A}_{\rho j} \subseteq \tilde{A}_{\rho^{\prime} j}\right)=p\left(\tilde{A}_{\rho^{\prime} j} \supseteq \tilde{A}_{\rho j}\right)=\frac{1}{2}\left(p^{-}\left(\tilde{A}_{\rho^{\prime} j} \supseteq \tilde{A}_{\rho j}\right)+p^{+}\left(\tilde{A}_{\rho^{\prime} j} \supseteq \tilde{A}_{\rho j}\right)\right) .
$$

Applying (P1.4) in Property 1, we obtain: $p^{-}\left(\tilde{A}_{\rho j} \supseteq \tilde{A}_{\rho^{\prime} j}\right)+p^{+}\left(\tilde{A}_{\rho^{\prime} j} \supseteq \tilde{A}_{\rho j}\right)=1$ and $p^{-}\left(\tilde{A}_{\rho^{\prime} j} \supseteq \tilde{A}_{\rho j}\right)+p^{+}\left(\tilde{A}_{\rho j} \supseteq \tilde{A}_{\rho^{\prime} j}\right)=1$. It is proven that:

$$
\begin{aligned}
& p\left(\tilde{A}_{\rho j} \supseteq \tilde{A}_{\rho^{\prime} j}\right)+p\left(\tilde{A}_{\rho j} \subseteq \tilde{A}_{\rho^{\prime} j}\right)= \\
& \frac{1}{2}\left(p^{-}\left(\tilde{A}_{\rho j} \supseteq \tilde{A}_{\rho^{\prime} j}\right)+p^{+}\left(\tilde{A}_{\rho j} \supseteq \tilde{A}_{\rho^{\prime} j}\right)\right)+\frac{1}{2}\left(p^{-}\left(\tilde{A}_{\rho^{\prime} j} \supseteq \tilde{A}_{\rho j}\right)+p^{+}\left(\tilde{A}_{\rho^{\prime} j} \supseteq \tilde{A}_{\rho j}\right)\right)= \\
& \frac{1}{2}\left(p^{-}\left(\tilde{A}_{\rho j} \supseteq \tilde{A}_{\rho^{\prime} j}\right)+p^{+}\left(\tilde{A}_{\rho^{\prime} j} \supseteq \tilde{A}_{\rho j}\right)\right)+\frac{1}{2}\left(p^{-}\left(\tilde{A}_{\rho^{\prime} j} \supseteq \tilde{A}_{\rho j}\right)+p^{+}\left(\tilde{A}_{\rho j} \supseteq \tilde{A}_{\rho^{\prime} j}\right)\right)=1 .
\end{aligned}
$$

Therefore, we prove that (P2.4) is valid. 


\section{Proof of (P2.5)}

If $p\left(\tilde{A}_{\rho j} \supseteq \tilde{A}_{\rho^{\prime} j}\right)=p\left(\tilde{A}_{\rho j} \subseteq \tilde{A}_{\rho^{\prime} j}\right)$, it is easily observed that $p\left(\tilde{A}_{\rho j} \supseteq \tilde{A}_{\rho^{\prime} j}\right)=p\left(\tilde{A}_{\rho j} \subseteq \tilde{A}_{\rho^{\prime} j}\right)=$ 0.5 according to (P2.4) as above. Thereby, (P2.5) is valid.

\section{Proof of (P2.6)}

Assume the contrary: $p\left(\tilde{A}_{\rho j} \supseteq \tilde{A}_{\rho " j}\right) \geq 0.5$ and but not. Then,

$$
p\left(\tilde{A}_{\rho j} \supseteq \tilde{A}_{\rho^{\prime} j}\right)<0.5
$$

holds.

When $1-\mu_{\rho j}^{+}-v_{\rho " j}^{+}<0$, we have $p^{+}\left(\tilde{A}_{\rho j} \supseteq \tilde{A}_{\rho^{\prime \prime} j}\right)=1$. According to (P1.1) in Property 1 , we know that $p^{-}\left(\tilde{A}_{\rho j} \supseteq \tilde{A}_{\rho^{\prime \prime} j}\right) \geq 0$, and thus, $p\left(\tilde{A}_{\rho j} \supseteq \tilde{A}_{\rho^{\prime \prime} j}\right) \geq 0.5$. In contrast, if $1-\mu_{\rho j}^{+}-v_{\rho " j}^{+} \geq 0$, the given assumption that $p\left(\tilde{A}_{\rho j} \supseteq \tilde{A}_{\rho^{\prime \prime} j}\right) \geq 0.5$ indicates that:

$$
\frac{1}{2}\left(p^{-}\left(\tilde{A}_{\rho j} \supseteq \tilde{A}_{\rho " j}\right)+p^{+}\left(\tilde{A}_{\rho j} \supseteq \tilde{A}_{\rho^{\prime \prime} j}\right)\right) \geq \frac{1}{2} \text {. }
$$

Therefore,

$$
p^{-}\left(\tilde{A}_{\rho j} \supseteq \tilde{A}_{\rho^{\prime \prime} j}\right)+p^{+}\left(\tilde{A}_{\rho j} \supseteq \tilde{A}_{\rho " j}\right) \geq 1 \text {. }
$$

Because $p^{-}\left(\tilde{A}_{\rho j} \supseteq \tilde{A}_{\rho^{\prime \prime} j}\right) \leq p^{+}\left(\tilde{A}_{\rho j} \supseteq \tilde{A}_{\rho " j}\right)$ using (P1.3) in Property 1, the necessary condition that $p\left(A_{\rho j} \supseteq A_{\rho " j}\right) \geq 0.5$ is as follows:

$$
p^{+}\left(\tilde{A}_{\rho j} \supseteq \tilde{A}_{\rho " j}\right) \geq \frac{1}{2} .
$$

Recall that $1-\mu_{\rho j}^{+}-v_{\rho " j}^{+} \geq 0$; thus,

$$
1-\frac{\left(1-v_{\rho " j}^{+}\right)-\mu_{\rho j}^{+}}{\left(1-\mu_{\rho j}^{+}-v_{\rho j}^{-}\right)+\left(1-\mu_{\rho " j}^{-}-v_{\rho " j}^{+}\right)} \geq \frac{1}{2} .
$$

It follows that:

$$
\frac{1-v_{\rho j}^{-}-\mu_{\rho " j}^{-}}{\left(1-v_{\rho j}^{-}-\mu_{\rho^{\prime \prime} j}^{-}\right)+\left(1-\mu_{\rho j}^{+}-v_{\rho^{\prime \prime} j}^{+}\right)} \geq \frac{1}{2} .
$$

Therefore, we obtain:

$$
0 \leq 1-\mu_{\rho j}^{+}-v_{\rho " j}^{+} \leq 1-v_{\rho j}^{-}-\mu_{\rho^{\prime \prime} j}^{-} .
$$

If $1-v_{\rho^{\prime} j}^{+}-\mu_{\rho^{\prime \prime} j}^{+}<0$, we have $p^{+}\left(\tilde{A}_{\rho^{\prime \prime} j} \supseteq \tilde{A}_{\rho^{\prime} j}\right)=1$. Therefore, $p\left(\tilde{A}_{\rho^{\prime \prime} j} \supseteq \tilde{A}_{\rho^{\prime} j}\right) \geq 0.5$ because $p^{-}\left(\tilde{A}_{\rho^{\prime \prime} j} \supseteq \tilde{A}_{\rho^{\prime} j}\right) \geq 0$. In contrast, if $1-v_{\rho^{\prime} j}^{+}-\mu_{\rho^{\prime \prime} j}^{+} \geq 0$, the given assumption that $p\left(\tilde{A}_{\rho^{\prime \prime} j} \supseteq \tilde{A}_{\rho^{\prime} j}\right) \geq 0.5$ indicates that:

$$
\frac{1}{2}\left(p^{-}\left(\tilde{A}_{\rho^{\prime \prime} j} \supseteq \tilde{A}_{\rho^{\prime} j}\right)+p^{+}\left(\tilde{A}_{\rho^{\prime \prime} j} \supseteq \tilde{A}_{\rho^{\prime} j}\right)\right) \geq \frac{1}{2},
$$

and

$$
p^{+}\left(\tilde{A}_{\rho^{\prime \prime} j} \supseteq \tilde{A}_{\rho^{\prime} j}\right) \geq \frac{1}{2} .
$$


In a similar way, we can obtain:

$$
0 \leq 1-\mu_{\rho^{\prime \prime} j}^{+}-v_{\rho^{\prime} j}^{+} \leq 1-v_{\rho^{\prime \prime} j}^{-}-\mu_{\rho^{\prime} j}^{-} \text {. }
$$

Consider the given assumption that $p\left(\tilde{A}_{\rho j} \supseteq \tilde{A}_{\rho " j}\right) \geq 0.5$. According to (P2.4) in Property 2, we know that $p\left(\tilde{A}_{\rho j} \supseteq \tilde{A}_{\rho^{\prime \prime} j}\right)+p\left(\tilde{A}_{\rho j} \subseteq \tilde{A}_{\rho^{\prime \prime} j}\right)=1$. Because $p\left(\tilde{A}_{\rho j} \supseteq \tilde{A}_{\rho^{\prime \prime} j}\right) \geq 0.5$, we obtain $p\left(\tilde{A}_{\rho j} \subseteq \tilde{A}_{\rho " j}\right)=p\left(\tilde{A}_{\rho " j} \supseteq \tilde{A}_{\rho j}\right) \leq 0.5$. When $1-v_{\rho j}^{+}-\mu_{\rho^{\prime \prime} j}^{+}<0$, we have $p^{+}\left(\tilde{A}_{\rho " j} \supseteq \tilde{A}_{\rho j}\right)=1$, which conflicts with $p\left(\tilde{A}_{\rho " j} \supseteq \tilde{A}_{\rho j}\right) \leq 0.5$. Therefore, it is reasonable that $1-v_{\rho j}^{+}-\mu_{\rho " j}^{+} \geq 0$. Accordingly, the sufficient condition that $p\left(\tilde{A}_{\rho " j} \supseteq \tilde{A}_{\rho j}\right) \leq 0.5$ is as follows:

It follows that:

$$
p^{+}\left(\tilde{A}_{\rho " j} \supseteq \tilde{A}_{\rho j}\right) \leq \frac{1}{2} .
$$

$$
1-\frac{\left(1-v_{\rho j}^{+}\right)-\mu_{\rho " j}^{+}}{\left(1-\mu_{\rho^{\prime \prime} j}^{+}-v_{\rho^{\prime \prime} j}^{-}\right)+\left(1-\mu_{\rho j}^{-}-v_{\rho j}^{+}\right)} \leq \frac{1}{2} .
$$

Therefore, we obtain:

$$
\frac{1-\mu_{\rho j}^{-}-v_{\rho^{\prime \prime} j}^{-}}{\left(1-\mu_{\rho j}^{-}-v_{\rho^{\prime \prime} j}^{-}\right)+\left(1-\mu_{\rho " j}^{+}-v_{\rho j}^{+}\right)} \leq \frac{1}{2} .
$$

Because $1-\mu_{\rho j}^{-}-v_{\rho^{\prime \prime} j}^{-} \geq 1-\mu_{\rho j}^{+}-v_{\rho^{\prime \prime} j}^{+} \geq 0$, we have:

$$
0 \leq 1-\mu_{\rho j}^{-}-v_{\rho " j}^{-} \leq 1-\mu_{\rho " j}^{+}-v_{\rho j}^{+} .
$$

Consider the given assumption that $p\left(\tilde{A}_{\rho^{\prime \prime} j} \supseteq \tilde{A}_{\rho^{\prime} j}\right) \geq 0.5$. We obtain $p\left(\tilde{A}_{\rho^{\prime} j} \supseteq \tilde{A}_{\rho^{\prime \prime} j}\right) \leq 0.5$ because $p\left(\tilde{A}_{\rho^{\prime \prime} j} \supseteq \tilde{A}_{\rho^{\prime} j}\right)+p\left(\tilde{A}_{\rho^{\prime} j} \supseteq \tilde{A}_{\rho^{\prime \prime} j}\right)=1$. When $1-v_{\rho^{\prime \prime} j}^{+}-\mu_{\rho^{\prime} j}^{+}<0$, we have $p^{+}\left(\tilde{A}_{\rho^{\prime} j} \supseteq \tilde{A}_{\rho^{\prime \prime} j}\right)=1$, which conflicts with $p\left(\tilde{A}_{\rho^{\prime} j} \supseteq \tilde{A}_{\rho^{\prime \prime} j}\right) \leq 0.5$. Therefore, the condition that $1-v_{\rho^{\prime \prime} j}^{+}-\mu_{\rho^{\prime} j}^{+} \geq 0$ is valid. The sufficient condition of $p\left(\tilde{A}_{\rho^{\prime} j} \supseteq \tilde{A}_{\rho^{\prime \prime} j}\right) \leq 0.5$ is as follows:

$$
p^{+}\left(\tilde{A}_{\rho^{\prime} j} \supseteq \tilde{A}_{\rho^{\prime \prime} j}\right) \leq \frac{1}{2} \text {. }
$$

In a similar way, we can obtain:

$$
0 \leq 1-\mu_{\rho^{\prime \prime} j}^{-}-v_{\rho^{\prime} j}^{-} \leq 1-\mu_{\rho^{\prime} j}^{+}-v_{\rho^{\prime \prime} j}^{+} .
$$

Summing the inequalities from (A7)-(A10), we obtain:

$$
\begin{gathered}
0 \leq 1-\mu_{\rho j}^{+}-v_{\rho^{\prime \prime} j}^{+}+1-\mu_{\rho^{\prime \prime} j}^{+}-v_{\rho^{\prime} j}^{+}+1-\mu_{\rho j}^{-}-v_{\rho^{\prime \prime} j}^{-}+1-\mu_{\rho^{\prime \prime} j}^{-}-v_{\rho^{\prime} j}^{-} \leq \\
1-v_{\rho j}^{-}-\mu_{\rho^{\prime \prime} j}^{-}+1-v_{\rho^{\prime \prime} j}^{-}-\mu_{\rho^{\prime} j}^{-}+1-\mu_{\rho^{\prime \prime} j}^{+}-v_{\rho j}^{+}+1-\mu_{\rho^{\prime} j}^{+}-v_{\rho^{\prime \prime} j}^{+} .
\end{gathered}
$$

It follows that:

$$
1-\mu_{\rho j}^{+}-v_{\rho^{\prime} j}^{+}+1-\mu_{\rho j}^{-}-v_{\rho^{\prime} j}^{-} \leq 1-v_{\rho j}^{-}-\mu_{\rho^{\prime} j}^{-}+1-v_{\rho j}^{+}-\mu_{\rho^{\prime} j}^{+} .
$$


Notice that:

$$
1-\mu_{\rho j}^{+}-v_{\rho^{\prime} j}^{+}+1-\mu_{\rho j}^{-}-v_{\rho^{\prime} j}^{-} \geq 1-\mu_{\rho j}^{+}-v_{\rho^{\prime} j}^{+}+1-\mu_{\rho j}^{+}-v_{\rho^{\prime} j}^{+}=2 \cdot\left(1-\mu_{\rho j}^{+}-v_{\rho^{\prime} j}^{+}\right),
$$

and

$$
1-v_{\rho j}^{-}-\mu_{\rho^{\prime} j}^{-}+1-v_{\rho j}^{+}-\mu_{\rho^{\prime} j}^{+} \leq 1-v_{\rho j}^{-}-\mu_{\rho^{\prime} j}^{-}+1-v_{\rho j}^{-}-\mu_{\rho^{\prime} j}^{-}=2 \cdot\left(1-v_{\rho j}^{-}-\mu_{\rho^{\prime} j}^{-}\right) \text {. }
$$

Therefore, the inequality in (A11) leads to:

$$
2 \cdot\left(1-\mu_{\rho j}^{+}-v_{\rho^{\prime} j}^{+}\right) \leq 2 \cdot\left(1-v_{\rho j}^{-}-\mu_{\rho^{\prime} j}^{-}\right) .
$$

It is easily observed that:

$$
1-\mu_{\rho j}^{+}-v_{\rho^{\prime} j}^{+} \leq 1-v_{\rho j}^{-}-\mu_{\rho^{\prime} j}^{-} .
$$

If $1-\mu_{\rho j}^{+}-v_{\rho^{\prime} j}^{+} \geq 0$, then we have:

$$
\left(1-v_{\rho j}^{-}-\mu_{\rho^{\prime} j}^{-}\right)+\left(1-\mu_{\rho j}^{+}-v_{\rho^{\prime} j}^{+}\right) \leq 2 \cdot\left(1-v_{\rho j}^{-}-\mu_{\rho^{\prime} j}^{-}\right) .
$$

The inequality above can be rewritten as follows:

$$
\frac{1-v_{\rho j}^{-}-\mu_{\rho^{\prime} j}^{-}}{\left(1-v_{\rho j}^{-}-\mu_{\rho^{\prime} j}^{-}\right)+\left(1-\mu_{\rho j}^{+}-v_{\rho^{\prime} j}^{+}\right)} \geq \frac{1}{2} .
$$

That is,

$$
1-\frac{\left(1-v_{\rho^{\prime} j}^{+}\right)-\mu_{\rho j}^{+}}{\left(1-\mu_{\rho j}^{+}-v_{\rho j}^{-}\right)+\left(1-\mu_{\rho^{\prime} j}^{-}-v_{\rho^{\prime} j}^{+}\right)} \geq \frac{1}{2},
$$

which implies that:

$$
p^{+}\left(\tilde{A}_{\rho j} \supseteq \tilde{A}_{\rho^{\prime} j}\right) \geq \frac{1}{2}
$$

If $1-\mu_{\rho j}^{+}-v_{\rho^{\prime} j}^{+}<0$, then we have $p^{+}\left(\tilde{A}_{\rho j} \supseteq \tilde{A}_{\rho^{\prime} j}\right)=1$. Therefore, the necessary condition that $p\left(\tilde{A}_{\rho j} \supseteq \tilde{A}_{\rho^{\prime} j}\right) \geq 0.5$ is satisfied.

Conversely, if $v_{\rho j}^{+}+\mu_{\rho^{\prime} j}^{+} \leq \mu_{\rho j}^{-}+v_{\rho^{\prime} j}^{-}$, we have:

$$
1-v_{\rho^{\prime} j}^{-}-\mu_{\rho j}^{-} \leq 1-\mu_{\rho^{\prime} j}^{+}-v_{\rho j}^{+} .
$$

Then, it follows that:

$$
2 \cdot\left(1-v_{\rho^{\prime} j}^{-}-\mu_{\rho j}^{-}\right) \leq\left(1-v_{\rho^{\prime} j}^{-}-\mu_{\rho j}^{-}\right)+\left(1-\mu_{\rho^{\prime} j}^{+}-v_{\rho j}^{+}\right) .
$$

The inequality above can be rewritten as follows:

$$
\frac{1-v_{\rho^{\prime} j}^{-}-\mu_{\rho j}^{-}}{\left(1-v_{\rho^{\prime} j}^{-}-\mu_{\rho j}^{-}\right)+\left(1-\mu_{\rho^{\prime} j}^{+}-v_{\rho j}^{+}\right)} \leq \frac{1}{2} .
$$


That is,

which implies that:

$$
1-\frac{\left(1-v_{\rho j}^{+}\right)-\mu_{\rho^{\prime} j}^{+}}{\left(1-\mu_{\rho^{\prime} j}^{+}-v_{\rho^{\prime} j}^{-}\right)+\left(1-\mu_{\rho j}^{-}-v_{\rho j}^{+}\right)} \leq \frac{1}{2},
$$

$$
p^{+}\left(\tilde{A}_{\rho^{\prime} j} \supseteq \tilde{A}_{\rho j}\right) \leq \frac{1}{2} \text {. }
$$

Therefore, the sufficient condition that $p\left(\tilde{A}_{\rho^{\prime} j} \supseteq \tilde{A}_{\rho j}\right) \leq 0.5$ is satisfied. When $v_{\rho j}^{+}+\mu_{\rho^{\prime} j}^{+}>\mu_{\rho j}^{-}+v_{\rho^{\prime} j}^{-}$, the necessary condition that $p\left(\tilde{A}_{\rho j} \supseteq \tilde{A}_{\rho^{\prime} j}\right) \geq 0.5$ is still satisfied. It is proven that $p\left(\tilde{A}_{\rho j} \supseteq \tilde{A}_{\rho^{\prime} j}\right) \geq 0.5$; this is a contradiction of (A6). Therefore, (P2.6) is valid.

Ting-Yu CHEN. She is currently a Professor of the Department of Industrial and Business Management and the Graduate Institute of Business and Management at Chang Gung University in Taiwan. She received her BS degree in Transportation Engineering and Management, MS degree in Civil Engineering, and $\mathrm{PhD}$ degree in Traffic and Transportation from National Chiao Tung University in Taiwan. She has published over 130 papers in peer-reviewed journals and 110 papers in conference proceedings. She has received a number of awards and grants. She is an Honorary Member of the Phi Tau Phi Scholastic Honor Society of Taiwan. Her current research interests include multiple criteria decision analysis, fuzzy decision-making methods and applications. 